\title{
Dynamic simulation of hydrodynamically interacting particles
}

\author{
By L. DURLOFSKY, J. F. BRADY \\ Chemical Engineering, California Institute of Technology, Pasadena, CA 91125, USA \\ AND G. BOSSIS \\ Laboratorie de Physique de la Matière Condensée, Université de Nice, Parc Valrose, \\ 06034 Nice Cedex, France
}

(Received 18 June 1986)

A general method for computing the hydrodynamic interactions among $N$ suspended particles, under the condition of vanishingly small particle Reynolds number, is presented. The method accounts for both near-field lubrication effects and the dominant many-body interactions. The many-body hydrodynamic interactions reproduce the screening characteristic of porous media and the 'effective viscosity' of free suspensions. The method is accurate and computationally efficient, permitting the dynamic simulation of arbitrarily configured many-particle systems. The hydrodynamic interactions calculated are shown to agree well with available exact calculations for small numbers of particles and to reproduce slender-body theory for linear chains of particles. The method can be used to determine static (i.e. configuration specific) and dynamic properties of suspended particles that interact through both hydrodynamic and non-hydrodynamic forces, where the latter may be any type of Brownian, colloidal, interparticle or external force. The method is also readily extended to dynamically simulate both unbounded and bounded suspensions.

\section{Introduction}

The behaviour of a finite number of small, hydrodynamically interacting particles, subject to externally imposed forces, torques or a linear shear flow, is fundamental to low-Reynolds-number hydrodynamies and finds application in a wide variety of systems such as suspensions, colloids and polymers, to name but a few. An adequate understanding of problems of this nature, however, has yet to be attained. In fact, the only exact, general solutions known to date are for two-body systems, particularly two rigid spheres. The many-body interaction problem, in this as in other branches of physics, poses an obstacle to further progress. Any theory that attempts to describe the dynamics of a system of particles suspended or dispersed in a fluid medium must address the issue of the hydrodynamic interactions among particles. For dilute systems, two-body interactions may suffice, but at even relatively low volume fractions of solids, many-body hydrodynamic interactions become important, affecting both quantitative and qualitative behaviour.

The purpose of this paper is to present a general simulation method capable of computing both static and dynamic properties of a finite, but arbitrarily large, system of hydrodynamically interacting particles, subject to prescribed forces, torques or an imposed linear shear flow, under conditions of vanishing particle Reynolds number. The method accounts for both near-field lubrication effects and the dominant 
many-body interactions. The method is fast, accurate and allows dynamic simulation of arbitrarily configured many-particle systems.

In the past, several methods concerned with hydrodynamically interacting, many-body systems have been developed. Kynch (1959) extended the method of reflections to account for effects from third and fourth bodies, showing that when relating the velocity disturbance of one sphere to the external force applied to another (i.e. the mobility interaction), third-body effects do not appear until $O\left(1 / r^{4}\right)$, where $r$ is a characteristic particle spacing, and fourth-body effects until $O\left(1 / r^{7}\right)$. Mazur \& Saarloos (1982) developed a Fourier-space multipole expansion method, which allowed them to calculate the sphere mobility functions for a finite system of spheres as a power series in inverse particle spacing. They showed that the dominant $n$-body mobility interaction appears at $O\left(r^{-3 n+5}\right)$, in agreement with the findings of Kynch. Mazur \& Saarloos calculated the sphere mobility functions up to $O\left(1 / r^{7}\right)$, including three- and four-body interactions. Though complex, their analysis is quite general and could, in theory, be extended to include higher-order many-body contributions. (Beenakker \& Mazur 1983 have included higher-order terms in the context of a suspension problem where they formally sum certain many-body interaction series.) S. Kim (1985, unpublished work), using a real-space multipole expansion method, solved the problem of three identical spheres located at the corners of an equilateral triangle sedimenting perpendicular to the plane of the triangle. He was able to obtain results for sphere centre-to-centre spacings of 2.5 radii or greater. He was, however, forced to extrapolate to obtain results at nearer spacings owing to the slow convergence of the series.

In addition to the analytical work discussed above, there have been several numerical studies of hydrodynamically interacting, many-body systems. Most notable is the work of Ganatos, Pfeffer \& Weinbaum (1978) in which they developed a collocation technique - expanding the solution to Stokes equations in the appropriate eigenfunctions and satisfying the boundary conditions at collocation points - to calculate particle velocities and drag coefficients for systems of identical spheres. Their method has only been applied to problems of high symmetry in order to reduce the number of unknowns, and under optimal conditions their method requires four boundary points per sphere; each boundary point has associated with it three unknowns. The location of the boundary points on the sphere surface is not, however, completely unambiguous. Ganatos et al. considered several static problems (i.e. instantaneous configurations) and some limited dynamics. The static problems involved calculation of the instantaneous velocities of spheres located in a straight chain, with the chain oriented either parallel or perpendicular to the direction of the imposed force. The number of spheres in a chain, as well as the spacing between spheres, was varied. They also computed the dynamic trajectories of several three-sphere sedimenting systems, following the sphere motions for hundreds of radii.

Other numerical methods could conceivably be applied to the many-sphere problem. Solution of the integral equation for Stokes flow, along the lines developed by Youngren \& Acrivos (1975), could be accomplished for many-body systems. This method has the advantage of being able to treat general particle shapes more easily than most other methods and is relatively simple to implement. In discretizing the particle surface, however, the number of unknowns per particle can become quite large, prohibiting its use for dynamic simulation. Finite-difference or finite-element schemes might also be applicable to systems of particles confined within boundaries, but they are not generally applicable to unconfined systems because the velocity 
disturbances caused by the particles decay so slowly that an extremely large computational domain would be required.

Though quite useful for some problems, all of the methods discussed above encounter serious difficulties when two or more particles come near contact. At close spacings, lubrication forces come into play, making actual physical contact of particles subjected to finite forces impossible. The force required to push two particles together diverges as the inverse of the spacing between the particle surfaces. These lubrication forces are not, however, included directly in any of the methods discussed above. In all of the analytic approaches, the lubrication forces must be built up by including many terms in the expansion in inverse particle spacing. This is a slowly convergent process; an infinite number of reflections is required to recover the lubrication forces between arbitrarily close particles.

The numerical method of Ganatos et al. experiences similar difficulties when spheres come near contact. For such configurations a greater number of boundary points, and thus more unknowns, is needed to satisfy the no-slip boundary conditions on the sphere surfaces. At a sphere centre-to-centre spacing of 2.005 radii, Ganatos et al. were forced to use 12 boundary points on each sphere, resulting in 36 unknowns per sphere. At closer spacings, which are likely to occur in a sufficiently complex system, even more boundary points would be required, resulting in prohibitive computer times and eliminating the possibility of computing dynamics. Direct solution of the integral equation encounters the same difficulty, with an increasing number of surface elements being required to resolve the lubrication-force densities. (It is possible, however, to devise a method that analytically takes lubrication explicitly into account in the integral formulation, thus keeping the number of unknowns per particle manageable (cf. the discussion in \$2).)

Bossis \& Brady (1984) developed a general molecular-dynamics-like method for simulating the dynamics of suspensions of hydrodynamically interacting particles. Though their work dealt with infinite systems, it is relevant to this discussion, particularly because Bossis \& Brady addressed the problem of directly including lubrication forces in the sphere-sphere interactions. In their simulation of a suspension of neutrally buoyant particles subjected to a linear shear flow Bossis \& Brady considered two different procedures for calculating particle interactions: pairwise additivity of velocities (mobilities) and pairwise additivity of forces (resistances), neither of which explicitly included many-body interactions. Using pairwise additivity of velocities, the mobility matrix (which relates sphere velocities to forces and torques) is formed directly; using pairwise additivity of forces, the resistance matrix (which relates forces and torques to velocities) is formed (cf. \$2). If forces and torques are prescribed, as they are in most problems, the former method is more convenient, because all that is required to determine the sphere velocities is a matrix multiplication. The latter method, by contrast, requires the solution of a system of equations. Bossis \& Brady found that lubrication forces, which kept particles from touching, were only preserved through pairwise addition of forces. In simulations that employed pairwise addition of velocities, particles freely touched and overlapped, unless strong, repulsive interparticle forces were introduced. Only by forming the resistance matrix, and then solving a system of equations to determine sphere velocities, were the spheres kept from touching.

As mentioned above, third-body effects do not appear in the mobility matrix until $O\left(1 / r^{4}\right)$. In the resistance matrix, by contrast, they appear at $O\left(1 / r^{2}\right)$. Thus, thirdand higher-body effects may be important to include when forming the resistance 
matrix directly. Bossis \& Brady neglected third- and higher-body interactions in their resistance formulation, with the physical assumption that, in the highly concentrated systems they studied, lubrication forces dominate and third-body interactions should be of much less importance.

From the above discussion it is clear that lubrication forces are preserved via pairwise additivity of forces (i.e. through directly forming the resistance matrix), but that three- and higher-body effects are more important to include in the resistance matrix than in the mobility matrix. Thus, using only pairwise additivity, it seems that the mobility formulation is more accurate for systems with widely spaced particles, but the resistance formulation is more accurate for closely spaced particles. In a general system of interacting particles, however, there are regions both of high concentration, where particles are near contact, and low concentration, where particles are widely spaced. A general technique must be able to describe the motion of the particles in such a system for any possible configuration.

The method that we shall present accounts for both the many-body interactions and the lubrication forces in a consistent, unambiguous way. It is fast, efficient, accurate, and can handle any configuration of a finite system of $N$ particles. The method is also readily extended to treat infinite suspensions. For simplicity, it is assumed that the particles are identical spheres, though the method could easily be adapted to include spheres of unequal sizes, and with a bit more difficulty it could be extended to particles of arbitrary size and shape. We shall develop two different versions of our method, each representing a different level of approximation. Each is therefore best suited to a particular class of problems. The first is most applicable to problems in which forces and torques are prescribed, but there is no linear shear flow. The second, more accurate, version is applicable to all problems, including those in which a linear shear flow is imposed; it does, however, require more computation time.

In brief overview, in our general method we first form the $N$-sphere mobility matrix, up to a particular order in inverse particle spacing depending on the version. This is accomplished by expanding the integral formulation for Stokes flow for the $N$-sphere system, in conjunction with Faxén laws for the particle velocities, in the moments of the force distribution on the surface of each particle. The first version, called the $\mathrm{F}-\mathrm{T}$ version, includes only two-body force/torque - translational velocity/angular velocity interactions. The second version, denoted $\mathrm{F}-\mathrm{T}-\mathrm{S}$, includes two-body force/torque/stresslet-translational velocity/angular velocity/rate of strain interactions. Because it includes only two-body interactions, the F-T mobility matrix only includes terms up to and including $O\left(r^{-3}\right)$. In the F-T-S version, the stresslet interactions incorporate all terms of $O\left(r^{-5}\right)$. In both versions, however, the $N$-sphere mobility matrix is a far-field approximation; no lubrication behaviour is included.

Once the $N$-sphere mobility matrix is formed, it is inverted, yielding a far-field approximation to the resistance matrix. Even though the mobility matrix only includes two-body mobility interactions, its invert includes many-body resistance interactions. Upon inversion, all many-body reflections of the mobility elements are performed, resulting in a resistance matrix that is a true many-body function; it is by no means a pairwise additivity of interactions. We shall show explicitly in the next section precisely which many-body reflections are included in each method. This resistance matrix, however, still lacks lubrication interactions. Lubrication is introduced in a pairwise additive manner, using the exact two-body resistance functions calculated by Arp \& Mason (1977), Jeffrey \& Onishi (1984) and Kim \& Mifflin (1985). 
In adding the resistance functions, care is taken to ensure that two-body interactions are not counted twice. The resistance matrix now includes near-field lubrication forces as well as far-field many-body interactions. Particle velocities can then be determined by solving a matrix equation.

In $\$ 2$ we formulate our simulation method for a finite system of $N$ spheres. Starting from the integral formulation for Stokes flow, we form the $N$-sphere mobility matrix as a moments expansion, and describe how to adjust its invert for lubrication. We also discuss in detail the precise nature of the many-body interactions that are included in the mobility invert, showing that both the screening characteristic of a porous medium and the effective viscosity of a viscous suspension are reproduced. In $\$ 3$ we compare some of our sedimentation results with those of Ganatos et al. and Kim. In all cases, agreement is excellent - typically three-significant-figure accuracy. It is also shown that our results for long chains of spheres, both sedimenting and immersed in a linear shear flow, are in accord with slender-body theory (Batchelor $1970 b$; Chwang \& Wu 1975). We shall also show some specific examples in which a simple pairwise additivity of mobility interactions leads to completely incorrect physics. Next, we present sedimentation and shear-flow results for problems not previously studied. Among these are dynamic trajectories that display interesting periodic behaviour; for example, it is shown that spheres initially oriented at the corners of a cube, with a prescribed force in a direction perpendicular to one of the cube faces, will continually invert themselves, reforming their initial configurations at later times. Finally, in $\$ 4$ we indicate the wide variety of finite particle systems that may now be studied rigorously, e.g. colloidal or cellular aggregates, micromechanical models of polymers, mobility of bioproteins, etc. We also discuss how the method we have developed may be generalized to non-spherical particles, to include particle interactions with solid boundaries and to dynamically simulate unbounded suspensions.

\section{Method}

We consider a finite system of rigid particles, small enough such that the particle Reynolds number, $U a / \nu$, where $U$ is a characteristic velocity, $a$ a characteristic size of the particles and $\nu$ the kinematic viscosity of the fluid, is much less than unity. The particles are suspended in an unbounded Newtonian fluid, which may be undergoing an imposed linear shear flow. Our intent is to develop a method that will allow us to calculate the translational and angular velocities of all the particles given the force and torque acting on each one.

In the absence of a shear flow, the basic problem is to generate an $N$-particle mobility matrix $\boldsymbol{M}$ that relates particle velocities to forces and torques:

$$
\boldsymbol{U}=\boldsymbol{M} \cdot \boldsymbol{F},
$$

where $U$ is the translational-angular velocity vector, $F$ is the force-torque vector, both of dimension $6 N$, and the $6 N \times 6 N$ square matrix $M$ is called the mobility matrix. The inverse problem is to calculate the forces and torques given the particle velocities:

$$
\boldsymbol{F}=\boldsymbol{R} \cdot \boldsymbol{U}
$$

where $\boldsymbol{R}$ is the resistance matrix and is the inverse of $\boldsymbol{M}$ :

$$
\boldsymbol{R}=\boldsymbol{M}^{-1} \text {. }
$$


(As shown below, (2.1) and (2.2) require modification when a linear shear flow is imposed.) In Stokes-flow problems, $\boldsymbol{M}$ and $\boldsymbol{R}$ possess many important properties; most fundamentally, $\boldsymbol{M}$ and $\boldsymbol{R}$ depend only on the instantaneous configuration of the particles, not on the particle velocities (Happel \& Brenner 1965). Also, both $M$ and $\boldsymbol{R}$ are symmetric, as can be shown from the reciprocal theorem, and positive definite, due to the dissipative nature of the system. Both properties apply for all possible configurations of the $N$ particles.

\subsection{Expansion of the integral equation and formation of the grand mobility matrix}

To develop our method, we begin with the integral representation for the velocity field in Stokes flow. At any point in the fluid or within the rigid particles, the velocity is given by (Ladyzhenskaya 1963)

$$
u_{i}(x)=u_{i}^{\infty}(x)-\frac{1}{8 \pi \mu} \sum_{\alpha=1}^{N} \int_{S_{\alpha}} J_{i j}(x-y) f_{j}(y) \mathrm{d} S_{y},
$$

where $u_{i}^{\infty}(x)$ is the velocity field in the absence of the particles, $S_{\alpha}$ is the surface of particle $\alpha, y$ is the location on the particle surface, and $x$ is a field point in the fluid-particle continuum. $J_{i j}$ is the free-space Green function or propagator for Stokes flow, known alternatively as the Stokeslet or Oseen tensor, and is given by

$$
J_{i j}(r)=\frac{\delta_{i j}}{r}+\frac{r_{i} r_{j}}{r^{3}}
$$

where $r=x-y, r=|r| . f_{j}(y)$ is the force density at the point $y$ on the surface of the particle. The summation indicates that the integration is to be performed over all $N$ particle surfaces. The force density can be expressed in terms of the fluid stress tensor $\sigma_{j k}$ by

$$
f_{j}(y)=\sigma_{j k}(y) n_{k}(y),
$$

where $n_{k}(y)$ is the surface normal vector pointing into the fluid. We further write

$$
\begin{aligned}
& F_{i}^{\alpha}=-\int_{S_{\alpha}} f_{i}(y) \mathrm{d} S_{y}, \\
& L_{i}^{\alpha}=-\int_{S_{\alpha}} \epsilon_{i j k}\left(y_{j}-x_{j}^{\alpha}\right) f_{k}(y) \mathrm{d} S_{y},
\end{aligned}
$$

where $F_{i}^{\alpha}$ is the total force exerted by particle $\alpha$ on the fluid, and $L_{i}^{\alpha}$ is the total torque exerted by the particle on the fluid measured relative to the 'centre' of the particle $\left(\boldsymbol{x}^{\alpha}\right)$.

As mentioned in the Introduction it is possible to solve (2.4) numerically by dividing the surface of each particle into elements and then resolving the linear system of equations for the force densities $f_{j}$ subject to the total force and torque conditions (2.7). While straightforward, this direct resolution can become computationally expensive when particles come into close proximity because of the large number of surface elements required to resolve the singular force distributions associated with lubrication. If the surface of each particle is divided into $M$ elements, the number of unknowns for the $N$-particle system is $(3 M+6) N$ : three components of force density for each element, and the translational and angular velocity of the centre of each particle. Thus, since there can be as many as 12 near neighbours in three dimensions, the number of unknowns per particle can become prohibitively large, precluding the use of this method for anything but static configurations of a few particles. 
It is possible, however, to analytically include the singular force densities associated with lubrication directly in the integral equation, and thus reduce the required number of surface elements. This can be accomplished by writing $f_{j}=f_{j}^{\mathrm{s}}+f_{j}^{\mathrm{o}}$, where the singular part $f_{j}^{s}$ is known analytically and the order-one remainder $f_{j}^{o}$ can be found by solving a system of equations. To handle an arbitrary configuration of particles, the minimum number of elements $M$ is 12 (12 possible near neighbours) in three dimensions, and $M=6$ in two dimensions. Specific configurations may require fewer than 12 elements, but in concentrated suspensions close-packed arrangements occur frequently. Thus, the minimum number of unknowns or degrees of freedom per particle is $3 M+6=42$, resulting in $42 N$ simultaneous equations to solve (15N in two dimensions). The number of degrees of freedom with this method is still quite large, however, making large dynamic simulation costly. We have developed an alternative method that makes use of both mobility and resistance information and substantially reduces the number of unknowns, while preserving lubrication and including excellent many-body hydrodynamics.

Rather than resolving the integral equation, we expand (2.4) in moments about the centre, $x^{\alpha}$ of each particle:

$$
\begin{aligned}
u_{i}(x)-u_{i}^{\infty}(x)=-\frac{1}{8 \pi \mu} \sum_{\alpha=1}^{N} \int_{S_{\alpha}} J_{i j}\left(x-x^{\alpha}\right) f_{j}(y) \mathrm{d} S_{y} \\
\quad+\left.\int_{S_{\alpha}} \frac{\partial}{\partial y_{k}} J_{i j}(x-y)\right|_{y=x^{\alpha}}\left(y_{k}-x_{k}^{\alpha}\right) f_{j}(y) \mathrm{d} S_{y}+\ldots
\end{aligned}
$$

Equation (2.8) provides a representation for the velocity at any point in the fluid in terms of a multipole expansion, with the $n$th multipole moment of particle $\alpha$ given by

$$
Q_{i \ldots j}^{\alpha}=-\int_{S_{\alpha}}\left(y_{i}-x_{i}^{\alpha}\right)^{n} f_{j}(y) d S_{y}
$$

The monopole or zeroth moment of the force density corresponds to the total force $(2.7 a)$. The first moment or dipole has both symmetric and antisymmetric parts; the antisymmetric part is the total torque $(2.7 b)$, and the symmetric part is known as the stresslet $\$_{i j}^{\alpha}$ :

$$
S_{i j}^{\alpha}=-\frac{1}{2} \int_{S_{\alpha}}\left\{\left(y_{i}-x_{i}^{\alpha}\right) f_{j}+\left(y_{j}-x_{j}^{\alpha}\right) f_{i}-\frac{2}{3} \delta_{i j}\left(y_{k}-x_{k}^{\alpha}\right) f_{k}\right\} \mathrm{d} S_{y}
$$

The stresslet $S_{i j}^{\alpha}$ is, in general, traceless, a consequence of continuity.

After the force and torque, which are prescribed quantities, the higher multipole moments depend on the relative configuration of all $N$ particles - they are induced moments and must be found by solving a set of equations (see below). Depending on the level of accuracy desired, the multipole expansion can be truncated at any order, but to include the effects of lubrication, all moments are needed. Since we shall introduce lubrication through the resistance matrix, we truncate the expansion after the dipole terms with the exception of two higher multipole contributions that are not induced but result from the finite size of the particle.

A single isolated sphere of radius $a$ translating in an unbounded quiescent fluid creates a velocity disturbance that may be written as

$$
u_{i}^{\prime}(x)=\frac{1}{8 \pi \mu}\left(1+\frac{1}{6} a^{2} \nabla^{2}\right) J_{i j}\left(x-x^{\alpha}\right) F_{j}^{\alpha}
$$


The sphere acts as a point force $J_{i j}$ plus a quadrupole contribution $\frac{1}{6} a^{2} \nabla^{2} J_{i j}$, resulting from its finite size. Part of the quadrupole-moment force density in (2.9) is not induced by interactions with other particles, but is proportional to the total force $F_{j}^{x}$ and depends on the sphere size and shape. This can be easily seen by writing the quadrupole moment as $Q_{j k l}^{\alpha}=A_{j k l m} F_{m}^{\alpha}$, where the tensor $A_{j k l m}$ must be isotropic for spheres, and the quadrupole-moment propagator $\nabla_{k} \nabla_{l} J_{i j}$ reduces to $\nabla^{2} J_{i j}$. For a particle of arbitrary shape, the quadrupole moment will not simply reduce to a Laplacian of $J_{i j}$ and (2.11) will not terminate after this term. Similarly, a sphere immersed in a pure straining motion acts as a stresslet plus an octupole, creating a velocity disturbance

$$
\begin{aligned}
u_{i}^{\prime}(x) & =\frac{1}{8 \pi \mu}\left(1+\frac{1}{10} a^{2} \nabla^{2}\right) K_{i j k}\left(x-x^{\alpha}\right) S_{j k}^{\alpha}, \\
K_{i j k} & =\frac{1}{2}\left(\nabla_{k} J_{i j}+\nabla_{j} J_{i k}\right) .
\end{aligned}
$$

We shall include both of these finite-size quadrupoles and octupoles in our truncated moments expansion, so that the remaining multipole moments are all induced by particle interactions; they are the so-called irreducible moments.

Thus, the velocity at any point in the fluid may be expressed in terms of the forces, torques and stresslets exerted by the particles on the fluid as

$$
u_{i}(x)=u_{i}^{\infty}(x)+\frac{1}{8 \pi \mu} \sum_{\alpha}\left(1+\frac{1}{6} a^{2} \nabla^{2}\right) J_{i j} F_{j}^{\alpha}+R_{i j} L_{j}^{\alpha}+\left(1+\frac{1}{10} a^{2} \nabla^{2}\right) K_{i j k} S_{j k}^{\alpha}+\ldots,
$$

where the propagator for the torque $R_{i j}$ is known as a rotlet or couplet and is given by

$$
R_{i j}(r)=\epsilon_{i j k} \frac{r_{k}}{r^{3}}=\epsilon_{l k j} \frac{1}{4}\left(\nabla_{k} J_{i l}-\nabla_{l} J_{i k}\right)
$$

To determine the motion of a particle $\alpha$ immersed in the flow field given by (2.13) we make use of the Faxén formulae for spheres (Batchelor \& Green 1972; note the sign difference in the definition of $S_{i j}$ ),

$$
\begin{aligned}
U_{i}^{\alpha}-u_{i}^{\infty}\left(x^{\alpha}\right) & =\frac{F_{i}^{\alpha}}{6 \pi \mu a}+\left(1+\frac{1}{6} a^{2} \nabla^{2}\right) u_{i}^{\prime}\left(x^{\alpha}\right), \\
\Omega_{i}^{\alpha}-\Omega_{i}^{\infty} & =\frac{L_{i}^{\alpha}}{8 \pi \mu a^{3}}+\frac{1}{2} \epsilon_{i j k} \nabla_{j} u_{k}^{\prime}\left(x^{\alpha}\right), \\
-E_{i j}^{\infty} & =\frac{S_{i j}^{\alpha}}{\left(\frac{20}{3}\right) \pi \mu a^{3}}+\left(1+\frac{a^{2}}{10} \nabla^{2}\right) e_{i j}^{\prime}\left(x^{\alpha}\right) .
\end{aligned}
$$

Here, $u_{i}^{\prime}(x)$, is the disturbance velocity field in (2.13) caused by the other particles, i.e. other than particle $\alpha$ itself and other than the impressed flow $u_{i}^{\infty}$. $e_{i j}^{\prime}=\frac{1}{2}\left(\nabla_{j} u_{i}^{\prime}+\nabla_{i} u_{j}^{\prime}\right)$ is the rate of strain of the disturbance flow, $E_{i j}^{\infty}$ is the rate of strain of the imposed flow, and $\Omega_{i}^{\infty}$ is the vorticity of the imposed flow. All velocity disturbances are evaluated at the centre of particle $\alpha$. Because the particles are rigid, there is no rate of strain within the $\alpha$ particle; hence, the absence of a kinematical property associated with particle $\alpha$ on the left-hand side of $(2.15 c)$. Note the similarity of the Faxén formulae and the form of the multipole expansion in (2.13).

Writing an equation like (2.15) for each particle $\alpha$, we can construct a grand mobility matrix $\mathscr{M}$ relating the translational velocity/angular velocity/rate of strain 
of each particle relative to the impressed flow to the force/torque/stresslet of all $\mathrm{N}$ particles:

$$
\left[\begin{array}{c}
\boldsymbol{U}-\boldsymbol{U}^{\infty} \\
-\boldsymbol{E}^{\infty}
\end{array}\right]=\mathscr{M} \cdot\left[\begin{array}{l}
\boldsymbol{F} \\
\boldsymbol{S}
\end{array}\right],
$$

where $U-U^{\infty}$ is a vector of dimension $6 N$ containing the translational and angular velocities of all $N$ particles relative to the impressed flow, $-\boldsymbol{E}^{\infty}$ is a vector of dimension $9 N$ that repeats the impressed rate of strain for each particle - all particles experience the same imposed rate of strain, $F$ is a $6 N$ vector containing the force and torque exerted by the particles on the fluid, and $\boldsymbol{S}$ of dimension $9 N$ contains the particle stresslets. The grand mobility matrix $\mathscr{M}$ is symmetric and positive definite and may be conveniently partitioned into submatricies:

$$
\mathscr{M}=\left[\begin{array}{ll}
M_{U F} & M_{U S} \\
M_{E F} & M_{E S}
\end{array}\right],
$$

where the subscripts indicate the coupling of the various components. $\boldsymbol{M}_{U F}$ relates particle velocities to forces and is what was called the mobility matrix $\boldsymbol{M}$ in (2.1) (actually, it is an approximation to the true mobility matrix), $\boldsymbol{M}_{U S}$ relates velocities and stresslets, $M_{E F}$ the rate of strain and forces, and $M_{E S}$ relates the rate of strain to stresslets.

Since the grand mobility matrix in (2.16) has been truncated at the level of force dipoles or stresslets, there are 11 unknowns or degrees of freedom per particle: 3 translational and 3 rotational velocities and 5 independent components of the stresslet (recall that the stresslet is symmetric and traceless). We shall denote this approximation to $\mathscr{M}$ as $\mathscr{M}^{\propto}$. To include higher multipole moments, one simply extends the vector of force moments on the right-hand side by the irreducible quadrupole, octupole, etc. moments, while on the left-hand side, the kinematical vector is extended with zeros as all higher velocity gradients within the particles or from the imposed flow are zero. (Note, it is not meaningful to suppose that there is an imposed quadratic flow field, because the boundaries creating this flow will contribute to the motion of the particles to the same order as the curvature of the flow.) The detailed elements of $\mathscr{M}^{\infty}$ are constructed from (2.15) and (2.13) along with (2.14), (2.12) and (2.5); they are given explicitly in Appendix A using the notation of Jeffrey \& Onishi (1984) and Kim \& Mifflin (1985).

$\mathscr{M}^{\infty}$ is a far-field approximation to the interaction among particles and includes terms up to $O\left(r^{-5}\right)$, where $r$ is a characteristic interparticle spacing. The leading error comes from the neglected induced quadrupoles, which are $O\left(r^{-6}\right)-$ a force propagates as $r^{-1}$ and induces a quadrupole in a second particle of strength $r^{-3}$, and this propagates a quadrupolar velocity ficld that decays as $r^{-3}$, giving the $O\left(r^{-6}\right)$ error. This is the leading error to $\boldsymbol{M}_{U F}$. The errors to $\boldsymbol{M}_{U S}$ or $\boldsymbol{M}_{E F}$ and $\boldsymbol{M}_{E S}$ are smaller, being $O\left(r^{-7}\right)$ and $O\left(r^{-8}\right)$ respectively. As constructed $\mathscr{M}^{\infty}$ would appear not to contain any two- or many-body interactions, and as written it does not. However, because the stresslets are unknown, (2.16) must be solved as a system of equations to determine $\boldsymbol{U}$ and $\boldsymbol{S}$ in terms of the given forces and the imposed flow, and the resolution of this system will introduce many-body interactions. Before discussing the nature of these many-body interactions, we shall first discuss how to include lubrication by use of the resistance matrix. This is most easily described if we first work with the small (as opposed to grand) mobility matrix $\boldsymbol{M}_{U F}$ only and neglect, for the moment, the stresslets. 


\subsection{Adjustment for lubrication}

In the absence of an imposed linear shear flow $\left(E^{\infty}=0\right)$, the stresslets are all induced, and it would seem reasonable to develop a method specifically for problems when only forces and torques are applied, the $F-T$ version. To include lubrication, we first invert $\boldsymbol{M}_{U F}$ to obtain a far-field approximation to the resistance matrix defined in (2.2). Inverting the small mobility matrix has the effect of reflecting all force-velocity interactions among all particles. This can be seen by examining the interactions between just two particles.

An element of the resistance matrix $\boldsymbol{R}_{\alpha \beta}$ gives the force on particle $\alpha$ due to the velocity of particle $\beta$. If we move particle $\beta$ at a prescribed velocity it will create a velocity disturbance that decays as $r^{-1}$. This velocity disturbance will induce a force in particle $\alpha$ because it is fixed, and this force will in turn create a velocity disturbance that decays as $r^{-2}$ - induced force strength $r^{-1}$ times velocity field due to a point force. Upon arriving at the moving particle $\beta$, this velocity disturbance will induce a force in this particle, because we must change the force exerted on it to keep it moving at the prescribed velocity. The process of reflected interactions between $\alpha$ and $\beta$ continues to all orders in $r^{-1}$, forming an infinite series. Inverting the mobility matrix for these two particles with just the point-force interactions ( $J_{i j}$ only) sums this infinite series of reflections. In Appendix B we show explicitly the equivalence of inverting and summing for the interaction along the line of centres between two particles. With $N$ point-force particles, the infinite number of reflected interactions among all $N$ particles is summed.

Thus, the invert of $M_{U F}$ is a true many-body approximation to the resistance matrix $\boldsymbol{R}$ of (2.2). In fact, the mobility invert reproduces the screening characteristic of a porous medium. If we have a large system of $N$ particles and one particle is moved, the resultant force experienced by a distant particle decays more rapidly than $r^{-1}$, specifically as $r^{-3}$ in three dimensions, because the interaction is screened by the intervening fixed particles (Howells 1974; Brinkman 1947). This screening is nothing more than the resultant of all the point-force reflections. Whatever elements are included in the mobility matrix - point force, finite size, stresslet interactions, etc. - upon inversion, reflections amongst all elements and all particles are summed.

This many-body approximation to the resistance matrix still lacks, however, lubrication. Lubrication would only be reproduced upon inversion of the mobility matrix if all multipole moments were included. To include lubrication, we introduce it in a pairwise additive fashion in the resistance matrix. For each element of $M_{U F}^{-1}$ we add the known exact two-sphere resistance interactions (Arp \& Mason 1977; Jeffrey \& Onishi 1984; Kim \& Mifflin 1985), which we shall designate as $\boldsymbol{R}_{2 \mathrm{~B}}$, for the two-body resistance matrix. However, part of the two-sphere resistance interactions, the far-field part, has already been included upon the inversion of $\boldsymbol{M}_{U F}$. Thus, in order not to count these interactions twice, we must subtract off the two-body reflected interactions already contained in $M_{U F}^{-1}$. The matrix composed of these two-body infinite reflection interactions is denoted by $\boldsymbol{R}_{2 \mathrm{~B}}^{\infty}$, and is found by simply inverting a two-body mobility matrix containing terms to the same order in $1 / r$ as $M_{U F}$. Thus, our approximation to the resistance matrix $\boldsymbol{R}$, which contains both near-field lubrication and far-field many-body interactions is

$$
\boldsymbol{R}=\boldsymbol{M}_{U F}^{-1}+\boldsymbol{R}_{2 \mathrm{~B}}-\boldsymbol{R}_{2 \mathrm{~B}}^{\infty}
$$


To determine the motion of the particles given the forces and torques acting on them, we solve the system of equations (2.2) with (2.18) as an approximation to the true $N$-particle resistance matrix.

We have designated the above procedure as the $\mathrm{F}-\mathrm{T}$ version, as only force-torque interactions were included. It will be shown in the next section that this method works well when there is no imposed linear shear flow. A more accurate version, and one that is necessary with an imposed shear flow, includes the stresslets directly in the grand mobility matrix ; our so-called F-T-S version. The invert of the grand mobility matrix in (2.16) is the grand resistance matrix $\mathscr{R}: \dagger$

$$
\left(\begin{array}{l}
F \\
\boldsymbol{S}
\end{array}\right)=\mathscr{R} \cdot\left(\begin{array}{c}
U-U^{\infty} \\
-\boldsymbol{E}^{\infty}
\end{array}\right)
$$

which may be partitioned as

$$
\mathscr{R}=\left[\begin{array}{ll}
\boldsymbol{R}_{F U} & \boldsymbol{R}_{F E} \\
\boldsymbol{R}_{S U} & \boldsymbol{R}_{S E}
\end{array}\right] .
$$

Note that $\boldsymbol{R}_{F U} \neq \boldsymbol{M}_{U F}^{-1}$, etc. $\boldsymbol{R}_{F U}$ is simply the matrix in the upper left-hand corner that relates $\boldsymbol{F}$ to $\boldsymbol{U}$ and is the same as $\boldsymbol{R}$ defined in (2.2). (At the level of interactions included, $\boldsymbol{R}_{F U}$ is an approximation to the exact $N$-particle $\boldsymbol{R}$.) Lubrication is included in the same manner as was done for the small resistance matrix, i.e.

$$
\mathscr{R}=\left(\mathscr{M}^{\infty}\right)^{-1}+\mathscr{R}_{2 \mathrm{~B}}-\mathscr{R}_{2 \mathrm{~B}}^{\infty}
$$

The motion of the particles with prescribed forces, torques and the linear shear flow is given by

$$
U-U^{\infty}=R_{F U}^{-1} \cdot\left[F+R_{F E}: E^{\infty}\right] .
$$

The F-T-S version is clearly more accurate than the $\mathrm{F}-\mathrm{T}$ version because more many-body reflections have been included in $\left(\mathscr{M}^{\infty}\right)^{-1}$. This additional accuracy has come at the cost of increasing the number of degrees of freedom per particle from 6 to 11 and, since inverting a matrix is an $N_{D}^{3}$ operation, where $N_{D}$ is the dimension of the matrix, results in an $\left(\frac{11}{6}\right)^{3}$ fold $(\approx 6.2)$ increase in computing time. The many-body interactions are, however, quite significant and, as we shall show, produce the effective viscosity of free suspensions.

To understand the nature of the many-body interactions, it is not necessary to include lubrication, and we can return to $(2.16)$ and $(2.17)$. If we resolve (2.16) for the unknown stresslets, we have

$$
\mathcal{S}=-M_{E S}^{-1}: M_{E F} \cdot F-M_{E S}^{-1}: E^{\infty}
$$

and the motion of the particles is given by

$$
U-U^{\infty}=\left(M_{U F}-M_{U S}: M_{E S}^{-1}: M_{E F}\right) \cdot F-M_{U S}: M_{E S}^{-1}: E^{\infty} .
$$

From (2.24) we see that by including the stresslets we approximate the small mobility matrix $M$ (defined in 2.1) by

$$
M=M_{U F}-M_{U S}: M_{E S}^{-1}: M_{E F}
$$

$\dagger$ Note, that because $S$ and $\boldsymbol{E}^{\infty}$ are symmetric and traceless, $\mathscr{R}$ as written contains redundant rows and columns and has no invert. The rows corresponding to $E^{\infty}$ must be written as $E_{x x}-E_{z z}$, $2 E_{x y}$, etc. before inversion. 
The physical significance of the second term is the following: just as $\boldsymbol{M}_{U F}^{-1}$ reflects all the force-velocity interactions, $\boldsymbol{M}_{E S}^{-1}$ reflects all the stresslet-rate-of-strain interactions. This 'dipole-dipole scattering' gives the first approximation to the effectivemedium behaviour of a free suspension. It is not difficult to show (Batchelor 1970a; Beenakker 1984) that for a force-free, torque-free suspension $(F=0)$, the average stress, and hence the effective viscosity, is given by an appropriate average of $\boldsymbol{M}_{E S}^{-1}$. Thus, this term represents the effective viscosity of the medium; it is actually the effective viscosity with local structure. The second term in (2.25) takes the force exerted by particle $\beta$, induces dipoles (stresslets) in all other particles $\boldsymbol{M}_{E F}$, which are dipole-dipole scattered from all particles in the system $\boldsymbol{M}_{E S}^{-1}$, i.e. they propagate through the effective medium, and end up incident on particle $\alpha, M_{U S}$. Note that the subscripts in (2.23)-(2.25) contract to give the sequence of scatterings.

Thus, we have devised a method that preserves lubrication forces by including interactions in the resistance matrix, and reproduces both the screening characteristic of a porous medium, $\boldsymbol{M}_{U F}^{-1}$, and the effective viscosity of free suspensions, $\boldsymbol{M}_{E S}^{-1}$. We shall show in the next section that this method, either the F-T version in the absence of an imposed shear flow, or the more accurate $\mathrm{F}-\mathrm{T}-\mathrm{S}$ version, gives excellent agreement with the available calculations for $N$ particles. Not surprisingly, there is an improvement in accuracy by going from the $\mathrm{F}-\mathrm{T}$ to the $\mathrm{F}-\mathrm{T}-\mathrm{S}$ version.

Two final remarks are in order. First, the approximation to the mobility and resistance matricies in the two versions preserves the positive definiteness of the matricies. Positive definiteness can easily be lost unless the many-body interactions are approximated properly. For example, if the F-T-S method is employed without the finite-size terms, i.e. the terms of $O\left(r^{-5}\right)$, positive definiteness can be lost.

And secondly, it may appear that the computation times for dynamic simulation are quite large owing to the necessity of inverting the grand mobility matrix at each time-step. However, significant changes in the grand mobility matrix only occur when the relative separation of two particles has changed by an amount comparable with the particle size. By contrast, the resistance matrix, which contains lubrication forces, changes on the scale of the lubrication forces, which vary with the separation of particle surfaces. Thus, there are two natural length (time) scales, and a multiple timescale method can be used. We only need invert (actually solve the equation set) the small resistance matrix $\boldsymbol{R}_{F U}$ in (2.22) each time-step; the grand mobility matrix $\mathscr{M}^{\infty}$ can be inverted infrequently - just how infrequently depends on the speed at which large-scale particle relative motion occurs.

\section{Results}

In this section we shall present static and dynamic simulation results for particles sedimenting in a quiescent fluid and for systems of neutrally buoyant particles immersed in a linear shear flow. To demonstrate the accuracy of our method we shall first compare some of our sedimentation results with those of Ganatos et al. (1978) and Kim (1985, unpublished work). Next, we shall present both static and dynamic results for other sedimenting systems that have not been previously considered. We shall also show that for linear chains of spheres our method reproduces slender-body theory. For groups of sedimenting particles the pairwise additivity of velocities approach can give reasonable estimates of the instantaneous particle velocities. In shear flow this is not the case, and we show by a simple example that incorrect physics are produced by the pairwise additivity of velocities. It is also shown that for particles 


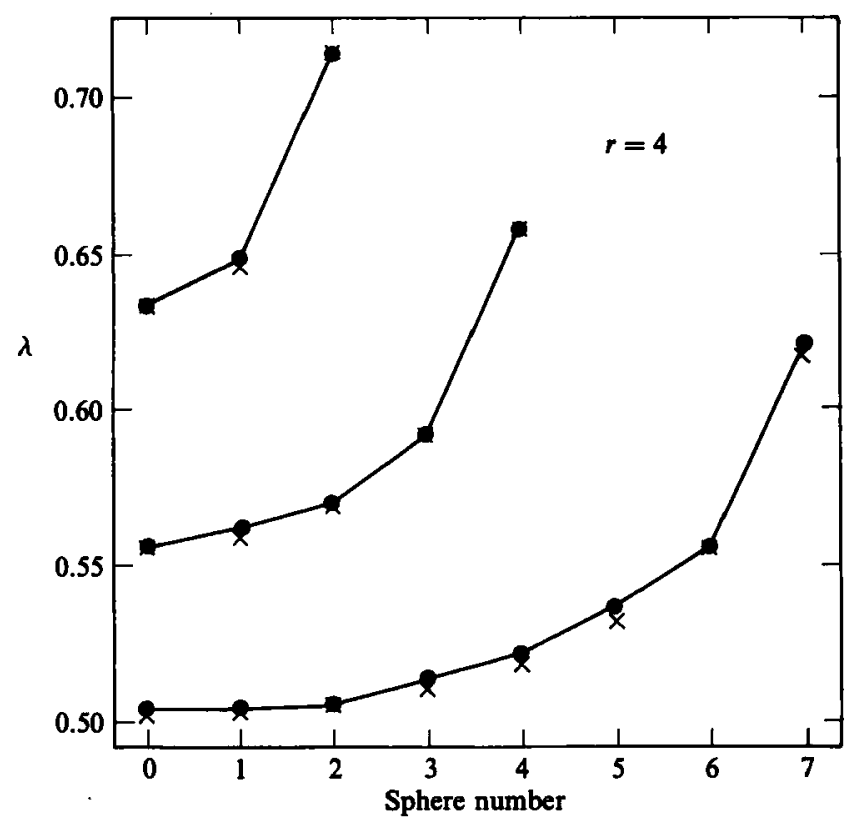

FigURe 1. Comparison of the drag coefficients $\lambda=F / 6 \pi \mu a U$ for horizontal chains of identical spheres sedimenting perpendicular to the line connecting their centres calculated using the F-T version $(x)$ with the numerical results of Ganatos et al. (1978) (O). The centre-centre sphere spacing $r$ is 4 radii. Results are shown for chains of 5, 9 and 15 spheres. Only half the chain is shown as the drag coefficient is symmetric about the central $(0)$ sphere.

undergoing Brownian motion the superposition of velocities leads to incorrect behaviour, whereas our method captures the proper physics.

\subsection{Sedimenting systems}

The first cases we consider are horizontal chains of spheres uniformly spaced at a centre-to-centre distance of four radii, sedimenting perpendicular to the line connecting the centres. The number of spheres in the chain is varied from three to fifteen. Because these sphere configurations are transitory, these results are instantaneous, applying only for the initial configuration. Figure 1 shows a comparison of our simulation results, represented as $x$ 's for the drag coefficient $\lambda$, defined as $\lambda=F / 6 \pi \mu a U$, where $F$ is the applied force, with the results of Ganatos et al. represented as solid circles, for five-, nine- and fifteen-sphere chains. These results were computed using the $\mathrm{F}-\mathrm{T}$ version described in $\$ 2$, though the $\mathrm{F}-\mathrm{T}-\mathrm{S}$ version gives identical results, owing to the relatively large interparticle spacing. The solid line connects spheres in the same chain. Results are shown only for half of the spheres in the chain because, for chains with odd numbers of spheres, the drag coefficient is symmetric about the central sphere and the angular velocity antisymmetric. Sphere number zero represents the central sphere.

The agreement obtained between the two methods is seen to be excellent. The largest discrepancy between any of the results displayed in figure 1 is less than $1 \%$. Although Ganatos et al. ascribe an uncertainty of only $0.02 \%$ to their drag-coefficient results for these configurations, an error of approximately $0.5 \%$ is introduced through the imprecision of reading the data from their plots. Thus, the less than $1 \%$ difference 


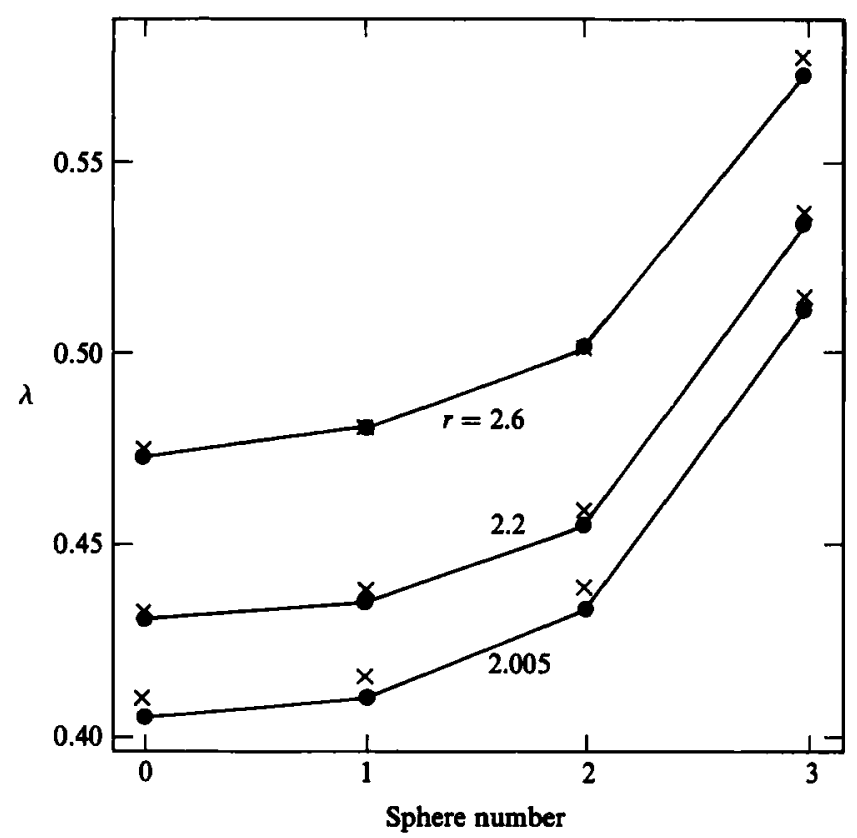

Figure 2. Comparison of the drag coefficient $\lambda=F / 6 \pi \mu a U$ for horizontal chains of seven sedimenting spheres calculated using the F-T version $(x)$ with the numerical results of Ganatos et al. (1978) (O). The sphere centre-centre spacing $r$ is varied: $r=2.6,2.2$ and 2.005 . Only half the chain is shown as $\lambda$ is symmetric about the central sphere.

between our results and theirs is of marginal significance. From figure 1, it is apparent that, as the number of spheres in the chain is increased, the spheres fall faster, with the central sphere falling considerably faster than the outer spheres. For a chain of fifteen spheres, the central sphere falls at nearly twice the speed of an isolated sphere, while the spheres on the ends fall at only about 1.6 times the speed of an isolated sphere.

Next, we consider sedimentation of horizontal chains of seven uniformly spaced spheres, with the dimensionless centre-to-centre distance $r(r$ is non-dimensionalized by the sphere radii) between spheres varied from 2.005 to 2.6. Again, these are instantaneous results. Figure 2 shows a comparison of our results using the F-T version, represented as $\times$ 's, with the results of Ganatos et al. represented as solid circles. The agreement is again excellent. Note that, as the spheres are positioned closer together, they fall faster. For these results, Ganatos et al. assign a maximum uncertainty of $0.4 \%$ to their $r=2.005$ drag-coefficient results. The maximum discrepancy between our results and theirs is about $1.5 \%$. Though not presented graphically, the maximum deviation between angular velocities at $r=2.005$ is $12 \%$. Ganatos et al. ascribe an uncertainty of $5 \%$ to their angular velocity results at this spacing. The discrepancies apparent between our simulation results and the collocation results of Ganatos et al., though small, can be reduced even further through the use of the F-T-S version. Table 1 shows a comparison of $\lambda$-and $\Omega$-values, calculated using the various methods, for a seven-sphere chain with a centre-to-centre spacing of 2.005. With the inclusion of stresslet interactions, the maximum discrepancy in $\lambda$ between our results and those of Ganatos et al. is reduced to about $0.6 \%$, while that for $\Omega$ is reduced to $10.6 \%$. 


\begin{tabular}{|c|c|c|c|c|}
\hline Sphere number & 0 & 1 & 2 & 3 \\
\hline$\lambda(\mathrm{F}-\mathrm{T})$ & 0.410 & 0.416 & 0.439 & 0.515 \\
\hline$\lambda(\mathbf{F}-\mathbf{T}-\mathrm{S})$ & 0.405 & 0.411 & 0.436 & 0.511 \\
\hline$\lambda$ (Ganatos et al. 1978) & 0.405 & 0.410 & 0.433 & 0.512 \\
\hline$\lambda(\mathbf{M})$ & 0.397 & 0.403 & 0.423 & 0.502 \\
\hline$\Omega(\mathrm{F}-\mathrm{T})$ & $\mathbf{0}$ & 0.0348 & 0.0974 & 0.219 \\
\hline$\Omega(\mathrm{F}-\mathrm{T}-\mathrm{S})$ & $\mathbf{0}$ & 0.0390 & 0.113 & 0.240 \\
\hline$\Omega$ (Ganatos et al. 1978) & $\mathbf{0}$ & 0.0352 & 0.111 & 0.231 \\
\hline$\Omega(\mathbf{M})$ & $\mathbf{0}$ & 0.0324 & 0.0865 & 0.278 \\
\hline
\end{tabular}

TABLE 1. Comparison of the results of various methods for a horizontal chain of seven nearly touching $(r=2.005)$ spheres sedimenting vertically

Table 1 also displays results that correspond to forming $M$ by pairwise additivity of velocities to the same order in $1 / r$ as the $F-T$ version, i.e. no adjustment for lubrication. These results (M) are in reasonable agreement with the results of the other methods : $\lambda$ deviates by at most $2 \%$ from Ganatos $e$ al., and $\Omega$ as much as $22 \%$. This simple method provides acceptable results in this case because there is no motion along the line of centres - spheres are not being forced toward each other - and lubrication forces are not needed. However, if one continued to use the superposition of velocities to follow the particle dynamics, aphysical results would soon arise, because motion along the line of centres develops quickly. Although not presented here, the drag coefficients for chains of particles falling vertically show equally good agreement with those calculated by Ganatos et al.

We next consider the problem treated by Kim (1985, unpublished work) - three identical spheres located at the verticies of an equilateral triangle of side $L$ falling in the direction perpendicular to the plane of the triangle. This configuration is a stable one, meaning that the spheres will remain at the verticies of the triangle for all time, and, in contrast to the chains of particles considered by Ganatos et al., which possessed planar symmetry, this problem is fully three-dimensional. Kim solved this problem using both the method of twin multipole expansions and the method of reflections. His results are strictly valid for sphere-sphere spacings of 2.5 radii or greater, as his series expansion converges slowly for closer particle spacings. Kim's results for the drag coefficient $\lambda$ are shown as the solid line in figure 3 , along with an extrapolated curve (dotted) calculated by Kim for closer particle spacings. The dashed line is the result one would obtain using a pairwise additivity of velocities, and shows a deviation of $16 \%$ at a separation of 2.5 . Our results using the F-T version are indicated by $x$ 's again, the agreement is quite good. The two results deviate slightly for $r<2.5$, with our results displaying a minimum in $\lambda$ at $r \approx 2.02$, while Kim's extrapolated results decrease monotonically. We believe the minimum is real, because for two spheres sedimenting perpendicular to the line joining their centres there is a minimum in $\lambda$ (maximum velocity) at $r \approx 2.01$ (Batchelor 1976). Thus, it seems reasonable that a minimum exists for groups of more than two spheres. $\dagger$

This is borne out by the next simplest stable arrangement of sedimenting particles (Hocking 1964)-spheres located at the corners of a square of side $L$ falling

$\dagger$ The lubrication approximations given by Jeffrey \& Onishi (1984) for the resistance functions $Y_{11}^{A}$, etc., which are accurate to $O\left(\xi \ln \xi^{-1}\right)$, where $\xi=r-2$, do not predict the minimum for two spheres. Only by extending the asymptotic formulae to terms of $O(\xi)$, whose coefficients we fit numerically, is the minimum calculated correctly. 


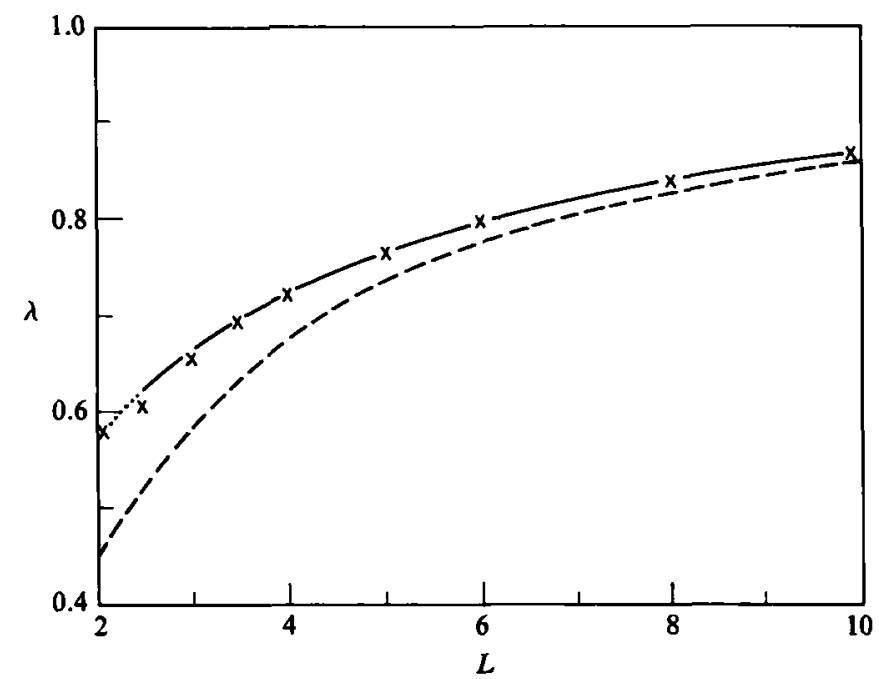

Figure 3. Comparison of the drag coefficient $\lambda=F / 6 \pi \mu a U$ for three identical spheres at the verticies of an equilateral triangle of side $L$ sedimenting perpendicular to the plane of the triangle. The $x$ are the results using the F-T version, the solid line is a theoretical calculation by Kim (1985, unpublished work). The dotted portion of this line for $L<2.5$ is an extrapolation of Kim's calculations; the series converges very slowly at these close separations. The dashed line is the drag coefficient predicted by pairwise additivity of interactions in the mobility matrix.

perpendicular to the plane of the square. Our results for the drag coefficient $\lambda$ using the F-T version are shown in figure 4. $\lambda$ is consistently smaller for four spheres than for three (compare figure 3 ), and we see the presence of a minimum at $L \approx 2.02$. As was the case for the equilateral triangle, all spheres rotate at the same speed, with each sphere rotating about a line that is perpendicular to the line connecting the sphere centre to the centre of the square. At $L=10, \Omega=0.0144$; at $L=2.5$, $\Omega=0.213$; at $L=2.1, \Omega=0.244$ (this is approximately the maximum); at $L=2.0001, \Omega=0.132$, and as $L \rightarrow 2, \Omega \rightarrow 0$ logarithmically. Similar behaviour will be observed for all regular polygons falling perpendicular to the plane containing the verticies. As discussed by Bretherton (1964), Hocking (1964) and Jayaweera, Mason \& Slack (1964) these configurations are stable at zero Reynolds number.

Another example of a static configuration of particles that provides a good check on the accuracy of our method is to examine the approach of the drag on a chain of spheres to that predicted by slender-body theory. For slender particles of spheroidal shape translating at constant velocity, Chwang \& Wu (1975) give the following expressions for the force or drag coefficients $C_{F_{1}}$ and $C_{F_{2}}$ :

$$
\begin{aligned}
& C_{F_{1}}=\frac{2}{3} \frac{1}{\ln (2 l / a)-\frac{1}{2}}\left[1+O\left(\frac{a}{l}\right)^{2}\right], \\
& C_{F_{2}}=\frac{4}{3} \frac{1}{\ln (2 l / a)+\frac{1}{2}}\left[1+O\left(\frac{a}{l}\right)^{2}\right],
\end{aligned}
$$

where $a$ and $l$ are the half-lengths of the minor and major axis respectively; $a / l \ll 1$. The drag coefficients $C_{F_{1}}$ and $C_{F_{2}}$ correspond to motion along and perpendicular to the major axis of the spheroid respectively. The force in each case has been nondimensionalized by $6 \pi \mu a U$. For a particle composed of $N$ spheres, $l / a=N$. Shown in table 2 are our calculated drag coefficients using the F-T-S version for chains of 


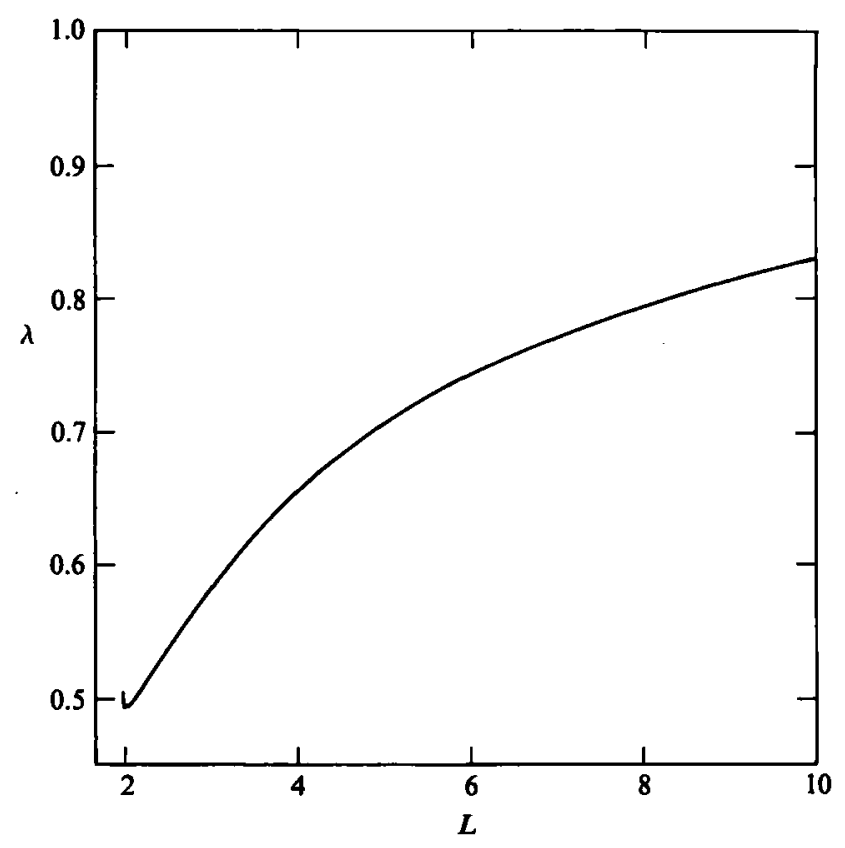

Figure 4. The drag coefficient $\lambda=F / 6 \pi \mu a U$ for four identical spheres at the corners of a square of side $L$ sedimenting perpendicular to the plane of the square calculated with the F-T version.

$N$ spheres at a centre-centre spacing of $2+10^{-5}$. The agreement is quite reasonable with a maximum deviation of $10 \%$. A large part of this deviation comes from the spheres at the end of the chain, and we also show the results for the drag coefficients when the contribution to the total drag from the spheres at either extremity is removed; the agreement improves considerably. Such good agreement is actually quite remarkable because a chain of spheres is neither a spheroidal particle, nor does it conform to the requirement of slender-body theory that the radius vary slowly along the particle axis. Examination of the local drag coefficients, i.e. for each sphere along the chain, shows a variation of roughly $\mathbf{4 0} \%$, with spheres near the centre having a drag coefficient lower than that given by slender-body theory, and spheres near the end having a larger drag coefficient. The proper $\ln (2 N)$ scaling of $(3.1)$ is, however, reproduced by our simulation method (cf. below for shear flow).

We have also computed some dynamic trajectories for sedimenting particles. Ganatos et al. made an extensive study of three spheres initially located on the same horizontal line falling under the action of gravity. Generally, our results agree well with theirs, with the exception that at long times the ultimate location of particles can be sensitive to specific earlier configurations. For example, in figure $5(a, b)$ we show the trajectories of three identical spheres sedimenting in the negative $y$-direction that were initially located at $y=0, x=-5,0$ and 7 , where the coordinates have been non-dimensionalized by the sphere radius. (This configuration corresponds to $C=1.4$ in the notation of Ganatos et al.) The time increment between successive points is 10 dimensionless time units (sphere radius over settling speed of an isolated sphere); the trajectories were terminated at a total time of $t=600$. The trajectories shown in figure $5(a)$ were calculated with the $\mathrm{F}-\mathrm{T}$ version and agree with those of Ganatos et al. until $t \approx 400(y \approx-525)$. At larger times the trajectories are quite different. Apparently, the trajectories are very sensitive to the sphere configurations in the time 


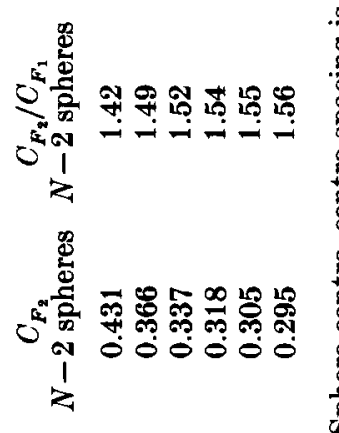

.

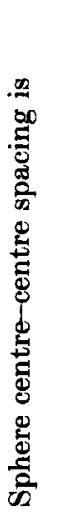

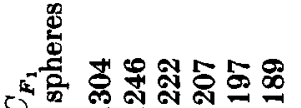

N

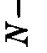

$\frac{2}{2}$

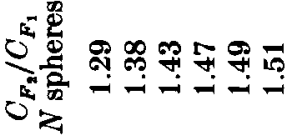

ติ

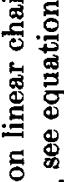

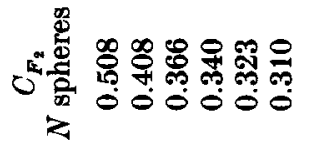

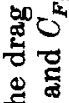

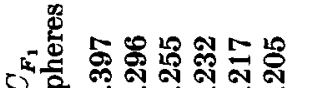
웅

:

起.

(

क्षे

$0^{-1}$

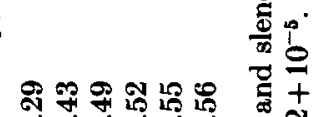

产

$\widehat{s}$

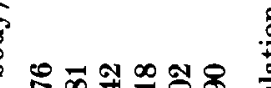

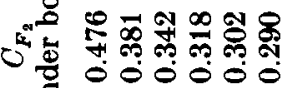
1 000000.

$\stackrel{\infty}{\infty}$

B

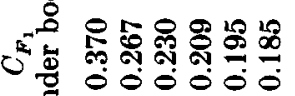

跑

乙 

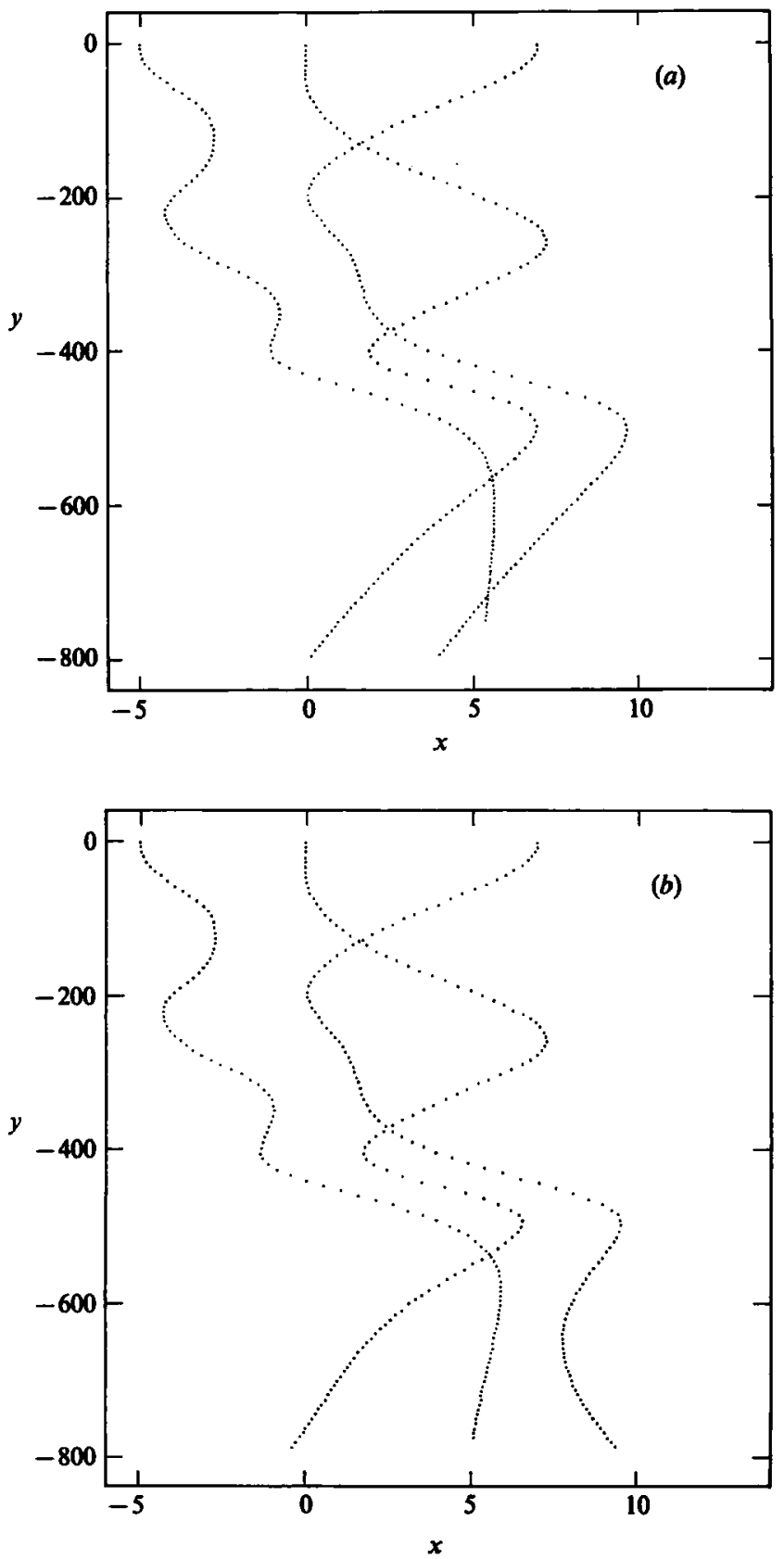

Fiaure 5. Trajectories of three identical sedimenting spheres. The spheres lie in the same plane with initial positions $y=0$ and $x=-5,0,+7$ based on sphere radius. The increment between dots is constant at 10 dimensionless time units. (a) Trajectories calculated with the $F-T$ version. (b) Same trajectories calculated with the more accurate $\mathbf{F}-\mathrm{T}-\mathrm{S}$ version. Note the different trajectories for $y<-500$, indicating a pronounced sensitivity to specific configurations. 


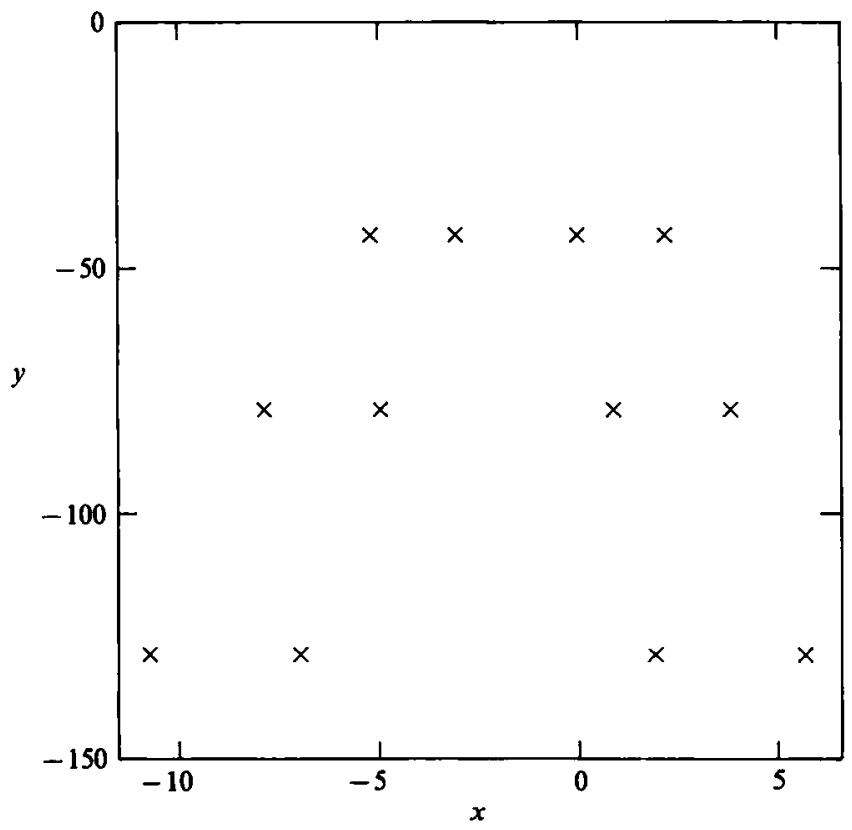

Figure 6 . The location of sphere centres that initially started at the corners of a square when they all lie in a line, calculated with the F-T version. The three sets of $x$ correspond to squares of side length $L=3$ (top row), 4 (middle row) and 5 (bottom row) respectively. The applied force is in the negative $y$-direction, parallel to the initial square side. The squares will periodically invert themselves as they fall.

interval $350<t<400$. A further indication of this sensitivity is shown in figure $5(b)$, where the same initial configuration is run using the $\mathbf{F}-\mathbf{T}-\mathbf{S}$ version. For times greater than $400(y<-525)$, completely different trajectories are observed - different from both the F-T results and the calculations of Ganatos et al. The sensitivity to specific configurations does not imply that our method is losing accuracy or fails to capture some physics. Rather, it is well known that dynamical systems with nonlinear interactions often display sensitivity, and such behaviour is to be expected. $\dagger$ The above example should not be interpreted as implying that after a certain time our results always deviate from those of Ganatos et al. Agreement or disagreement depends critically on the initial condition.

We next consider the problem, first studied by Hocking (1964), of four spheres located initially at the corners of a square of side $L$ with an applied force in the direction of one of the sides. As discussed by Hocking, this configuration is not stable and is actually periodic in time. Initially the top two spheres are drawn down and inward, and the bottom two move down and outward. This process continues until all spheres lie on the same line. Since this configuration has reflection symmetry upon both changing the sign of the imposed force $(F \rightarrow-F)$ and in the plane containing the force vector, the spheres will reform the square with the groups of upper and lower particles interchanged. This process will repeat indefinitely. The time required for the

$\dagger$ We also performed simulations with a version intermediate between $\mathrm{F}-\mathrm{T}$ and $\mathrm{F}-\mathrm{T}-\mathrm{S}$. Putting $M_{E S}$ diagonal propagates all dipole-dipole interactions through the pure fluid rather than the effective medium, and results with such a version are qualitatively the same as with the $\mathbf{F}-\mathrm{T}-\mathrm{S}$ version. The $x$-positions differ slightly from those in figure $5 b$, but the ordering of particles is the same. 


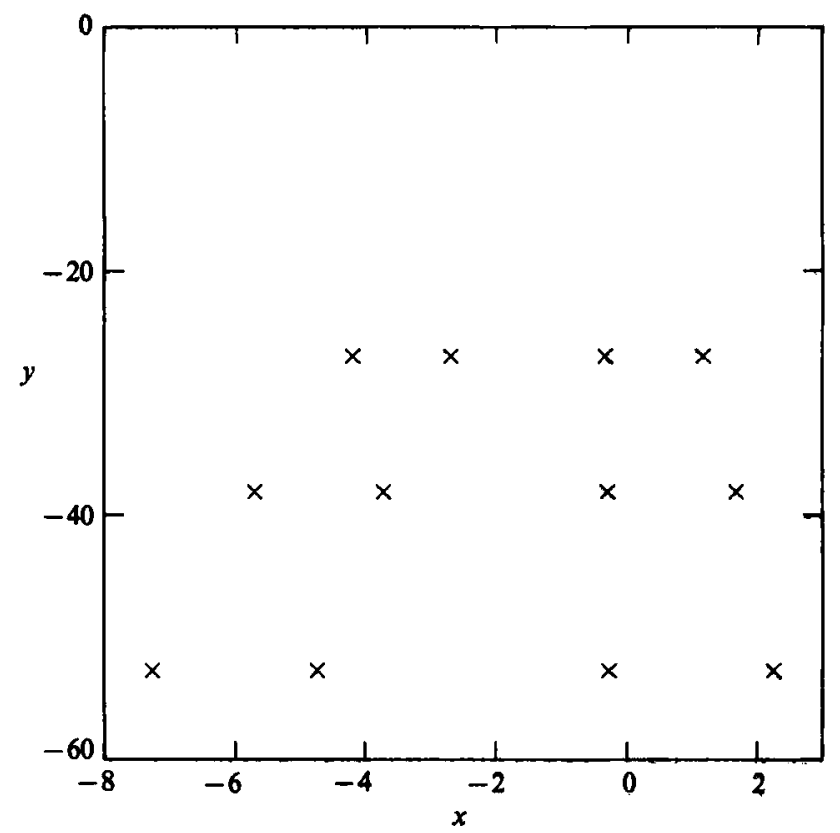

Froure 7. The location of sphere centres that initially started at the corners of a cube when they all lie in the same plane, calculated with the F-T version. The three sets of $\times$ correspond to oubes of side $L=3$ (top row), 4 (middle row) and 5 (bottom row) respectively. Only half the sphere centres are shown because of the reflectional symmetry in the $(y, z)$ - and $(y, x)$-planes; the applied force is in the negative $y$ direction. The cube configuration periodically inverts itself.

square to invert itself is twice the time required for the spheres to form a line after being initially placed at the corners of a square. Figure 6 shows the location of the centres of the spheres when they all lie on a line for square sides $L=3,4$ and 5 . The time required for complete inversion for the three cases is 45.4, 96.6 and $\mathbf{1 7 4 . 2}$ respectively; the time increases considerably as the size of the square increases. (Note, not all positions of spheres in a line that are reflectionally symmetric about the plane containing the force, i.e. symmetric in $x$ in figure 6, will produce periodic trajectories.) These results were calculated using the F-T version. With the F-T-S version, the particle velocities are slightly smaller, and the period for inversion with $L=3$ increases to $\mathbf{4 5 . 4}$ from $\mathbf{4 8 . 4}$.

Similar behaviour is observed for spheres placed at the corners of a oube of side $L$. The top four spheres all move inward, towards the centre of the cube and the bottom four outward as the group falls, until at one time all spheres lie in the same plane. We now have three-fold reflectional symmetry about three perpendicular planes - two parallel and one perpendicular to the direction of the applied force. Shown in figure 7 are the centres of half the spheres when they all lie in the $(y, z)$-plane, for three cubes $L=3,4$ and 5 , computed with the F-T version. Only the $x$-locations of half the sphere centres need be given because of the symmetry of the configuration. The periods for inverting the cube are: $19.3,32$ and 50.2 respectively, considerably shorter than for a square. This inversion process is not necessarily limited to squares and cubes. Other regular polyhedrons may display similar behaviour.

The above-calculated dynamics are quite stable in a numerical sense. Inverting squares were run for several periods $(\approx 10)$ and showed no loss of accuracy $(6$ significant figures). We tested the sensitivity to the initial configuration by perturbing 
a single sphere of a square of side 3 a small amount - initial coordinate $(-0.01,0)$ rather than $(0,0)$. The square succeeded in maintaining the first inversion with the same $0(0.01)$ perturbation, but by the time of the second inversion for the unperturbed square, the square configuration is lost and the subsequent dynamics are completely different. This is another illustration (recall figure 5 for three spheres with the F-T and $\mathrm{F}-\mathrm{T}-\mathrm{S}$ versions) of the sensitivity of particle trajectories to initial conditions, suggesting, as one would expect, the possibility of chaotic behaviour for particle dynamics.

The last example of sedimenting systems is the dynamics of the horizontal chains discussed previously. We observed some rather interesting behaviour for chains with initial centre-centre spacing of 2.005 at $N=7,8,9$ and 11 spheres (the only cases examined). In all cases, the central $N-4$ spheres pulled away from the spheres at either end (cf. drag coefficient $\lambda$ in figure 1), leaving the four end spheres trailing in two separate groups. At long times the two spheres in each group may be in contact. The central $N-4$ spheres formed the following configurations: for $N=7$, a triangle, which only comes into full three-way contact for very long times, $t>200$; for $N=8$, a square, which periodically inverts itself; for $N=9$, a pentagonal cluster with each sphere in contact with two neighbours; and for $N=11$, a heptagonal cluster. It is not known whether this unusual behaviour persists for arbitrary $N$, nor the degree to which it depends on the initial separation between spheres.

\subsection{Sheared systems}

While the method that we have developed accurately reproduces both the drag coefficients for instantaneous configurations and the dynamics of sedimenting particle systems, the real power of the method to maintain lubrication forces has not been exploited. Indeed, for some simple instantaneous configurations of sedimenting particles, the superposition of velocities or pairwise additivity of mobility interactions works well (cf. table 1). For sheared systems, or when there is significant relative motion along the line of centres of particles, however, this is no longer true; even instantaneous results are in gross error and miss essential physics. We illustrate this by the following two examples.

Four identical spheres are placed in a line with a centre-centre spacing of $2+10^{5}$. The line joining the centres is oriented along the compressive axis of a simple shear flow. The two central particles, numbers 2 and 3, will move towards each other with relative velocities along the line of centres that should be small, i.e. the same order of magnitude as the surface separation $O\left(10^{-5}\right)$, because of lubrication. Using the F-T-S version, we find $U^{2}-U^{3}=-6.5 \times 10^{-5}$, which is not very different from the exact result for two isolated spheres of $-4 \times 10^{-5}$. There is a slight enhancement of the relative velocity of the central spheres caused by the two end spheres. The pairwise additivity of mobility interactions, however, gives an enormous relative velocity, $U^{2}-U^{3}=-1.9$, five orders of magnitude too large! Rather than moving very slowly toward one another, particles 2 and 3 would physically touch and overlap if there were no other repulsive forces present to prevent contact. The pairwise additivity of velocities completely misses the physics of particle interactions when there is significant relative motion along the line of centres between particles. (Note, for this small cluster, the F-T version gives essentially identical results.)

Similar aphysical behaviour is displayed by pairwise additivity of mobilities in a different context. To simulate the dynamics of particles subject to Brownian motion (cf. Bossis \& Brady 1987) requires the evaluation of the configuration space 


\begin{tabular}{|c|c|}
\hline$N$ & $S_{12} / S_{12}^{S B B}$ \\
\hline 5 & 1.10 \\
\hline 10 & 1.10 \\
\hline 15 & 1.04 \\
\hline 20 & 1.01 \\
\hline 25 & 0.998 \\
\hline 30 & 0.991 \\
\hline 40 & 0.990 \\
\hline 49 & 0.993 \\
\hline
\end{tabular}

TABLE 3. Comparison of slender-body theory and simulations for the stresslet of a linear chain of $N$ spheres. The sphere centre-centre spacing is $2+10^{-5}$. The theoretical formula for the stresslet is given in equation (3.2).

divergence of the mobility matrix (Batchelor 1976) $\nabla \cdot M=\nabla \cdot \boldsymbol{R}^{-1}$. For four particles aligned in a row and numbered $1,2,3$ and 4 , the velocity of each particle from $\nabla \cdot \boldsymbol{R}^{-1}$ is along the line of centres. With a centre-centre spacing of $2+10^{-3}$, the $\mathrm{F}-\mathrm{T}$ version gives: $U^{1}=-4.73, U^{2}=-1.61, U^{3}=1.61$ and $U^{4}=4.73$. Pairwise additivity in the mobility matrix gives: $U^{1}=-1.62, U^{2}=-0.022, U^{3}=0.022$ and $U^{4}=1.62$. While not as dramatic as the previous example, the differences are quite significant and have a profound effect on the dynamics of Brownian particles. As the chain of spheres increases in length, the superposition of velocities gives essentially zero relative velocity for central particles, with the two particles at the end moving as they would if they were the only two particles in the system. Two isolated spheres at a separation of $2+10^{-3}$ move in opposite directions with velocities of magnitude 1.61. The proper behaviour of a chain is completely different, with central particles flying apart at speeds of 1.61, and each additional particle in the chain adding approximately $2 \times 1.61=3.22$ in relative velocity. While this Brownian contribution does not lead to as catastrophic behaviour as in shear flow, i.e. particles will not overlap, the dynamics of suspensions of such particles would be fundamentally different.

Another example that demonstrates the accuracy of the simulation method for sheared systems is the approach to slender-body theory for linear chains. A chain of $N$ spheres almost in contact, i.e. a separation of $2+10^{-5}$, should act, at least instantaneously, as a rigid rod. For force- and torque-free spheroidal rods, the relevant quantity is the total stresslet that the rod exerts on the fluid. Batchelor $(1970 b)$ has shown that the component of the stresslet along the axis of the rod $S_{12}^{\text {sB }}$ is given by

$$
S_{12}^{\mathrm{SB}}=\frac{20}{3} \pi \mu a^{3} E_{12} \frac{1}{5} \frac{(l / a)^{3}}{\ln (2 l / a)-\frac{3}{2}}\left[1+O\left(\frac{a}{l}\right)^{2}\right]
$$

where $a$ and $l$ are the half-lengths of the minor and major axis, respectively, and $E_{12}$ is the component of the rate-of-strain tensor along the major axis. For a chain of $N$ spheres, $a$ is the radius and $N=l / a$. Table 3 shows a comparison of the total stresslet $S_{12}$, found by summing the stresslets of the individual spheres, and (3.2) for the slender-body prediction. There is a maximum deviation of $10 \%$ and a slight minimum that occurs for $N=37$. The reported results are for spheres that are individually fixed in a simple shear flow. At these close spacings identical results are obtained for freely suspended spheres. As the spacing between particles increases, the 
stresslets for fixed and free particles deviate. A similar calculation using the F-T version gave the proper scaling with sphere number, but the coefficient is uniformly smaller by roughly a factor of 2 , i.e. a $50 \%$ error.

\section{Conclusions}

The simulation method presented in this paper has been shown to provide accurate results for a variety of problems. The method is completely general - all configurations are treated in the same way; no special precautions need be taken to simulate the dynamics of widely spaced or near-contact arrangements of spheres. Both versions of the method reproduce the screening characteristic of a porous medium upon inversion of the mobility matrix, and the $\mathrm{F}-\mathrm{T}-\mathrm{S}$ version goes further in generating the effective medium behaviour of free suspensions. Accuracy improves from the F-T to F-T-S version, so in principle a tradeoff between accuracy and computation time can be made, although the effective medium aspect of the $\mathbf{F}-\mathbf{T}-\mathrm{S}$ version is a highly desirable component.

It seems appropriate at this point to comment on the amount of computer time required by the various versions of our simulation method. For each version, a matrix must be inverted and a matrix equation then solved. These matrix manipulations require $O\left(N^{3}\right)$ computer operations, while other aspects of the program, such as forming the mobility and resistance elements, require only $O\left(N^{2}\right)$ operations. For problems with planar symmetry, the $\mathrm{F}-\mathrm{T}$ version requires the inversion of a $3 N \times 3 N$ matrix and the solution of a $3 N$ system of equations, which amounts to $O\left(18 N^{3}\right)$ operations $\left(\frac{1}{2}(3 N)^{3}\right.$ for inversion plus $\frac{1}{6}(3 N)^{3}$ for solution), while for fully threedimensional problems, $O\left(144 N^{3}\right)$ operations are required. The F-T-S version with planar symmetry requires $O\left(112 N^{3}\right)$ and $O\left(702 N^{3}\right)$ operations in three dimensions. Recall, however, that the mobility matrix need not be formed frequently, so, at most timesteps, only a matrix solution of a smaller equation set, and not an inversion, need be performed.

To get an appreciation of what these operation requirements imply, let us compare the operation requirements of our method with those of the collocation method of Ganatos et al. (1978). For configurations with no near-touching spheres, their method requires four boundary points per sphere, which translates to 12 unknowns per sphere. Their method does not require a matrix inversion, only a solution. However, their matrix does not appear to be symmetric (they do not mention that it is), so $O\left(576 N^{3}\right)$ operations are required. Our F-T version represents a 32 -fold improvement in computer time, and our $\mathrm{F}-\mathrm{T}-\mathrm{S}$ version a five-fold improvement, over the collocation method of Ganatos et al. Much more substantial savings are achieved, however, when spheres come near contact. For such configurations, Ganatos et al. may require 12 boundary points, resulting in $O\left(15552 N^{3}\right)$ operations. Now, the F-T version offers an 864-fold reduction in computer time and the $\mathrm{F}-\mathrm{T}-\mathrm{S}$ version a 138-fold reduction. Calculations without planar symmetry were not performed by Ganatos et al, and it is not known what the computational demands are for a fully three-dimensional collocation method.

The operation requirements of the direct resolution of the integral equation for Stokes flow that analytically accounts for the singular force densities discussed in $\$ 2$ are $O\left(562 N^{3}\right)$ for planar symmetry and $O\left(12348 N^{3}\right)$ for fully three-dimensional systems. Here, we have assumed a minimum number of surface elements of 6 in two dimensions and 12 in three dimensions, corresponding to the maximum number of 
near neighbours. Our F-T-S version provides a 5 -fold and an 18 -fold saving in two and three dimensions respectively. The accuracy of this direct resolution method has not been fully tested, however, so the number of surface elements required can only be estimated. The above comparisons apply for instantaneous configurations only. In dynamic simulation both methods permit multiple time-stepping, and full comparison must await future trials.

The method that we have developed thus appears to be both accurate and computationally efficient. Although we have demonstrated the method with only a few examples it should be clear that a wide variety of problems may now be addressed. External forces other than gravity, as well as interparticle forces may be included with no modification to the method. The encouraging results with slender-body theory suggest that particles of complex shape may be modelled by groups of spheres. With interparticle forces these groups will be able to model particles with internal degrees of freedom, such as micromechanical models of polymers and, with the inclusion of Brownian motion as indicated in $\$ 3$ an even broader class of problems can be addressed. In short, we have developed a method to accurately dynamically simulate a finite number of spheres subject to hydrodynamic, external, interparticle and Brownian forces.

In the method that we have developed, we have only considered identical spheres, but the extension to a distribution of sphere sizes is straightforward. The only additional requirements are the hydrodynamic interactions between different-sized spheres, and these are available in the work of Jeffrey \& Onishi (1984). $†$ Non-spherical particles can be modelled by simply 'joining' spheres together as in the slender-body examples discussed in $\$ 3$. However, it may be possible to treat non-spherical particles directly, because the mobility matrix to the level of dipoles is not difficult to construct. In fact, for ellipsoidal particles all the required formulae are in the work of $\mathrm{Kim}$ (1986). Lubrication interactions for the resistance matrix are also available (Cox 1974). An approach such as this has the advantage over joining spheres together of reducing the number of degrees of freedom per particle and thus saving computation time.

Other extensions much more complex than the above are also possible. The method can be (and has been, cf. Bossis \& Brady 1984) easily modified to simulate infinite systems, i.e. suspensions. Additional issues arise here in the use of periodic boundary conditions and the non-convergent nature of the long-range hydrodynamic interactions, but these can be handled in a rigorous fashion (Brady \& Bossis 1985). Indeed, since the $\mathrm{F}-\mathrm{T} \rightarrow \mathrm{S}$ version reproduces the effective-medium behaviour, one is a long way towards a proper treatment of the many-body hydrodynamic interactions in suspensions. Finally, the method can also be extended to include the presence of solid boundaries (Durlofsky 1986), enabling the investigation of boundary effects in suspensions.

This work was supported in part by the National Science Foundation under grants PYI CBT-8451597 and INT-8413695 and by the Centre de Calcul Vectoriel pour la Recherche.

$\dagger$ Unfortunately, the resistance elements for the force-shear and stresslet-shear couplings $\left(\boldsymbol{R}_{F E}\right.$ and $\boldsymbol{R}_{S E}$ in $\left.(2,20)\right)$ for unequal spheres are not yet available. 


\section{Appendix A. Elements of the grand mobility matrix}

The grand mobility matrix $\mathscr{M}$ in (2.16) written in terms of individual elements in the notation of Jeffrey \& Onishi (1984) and Kim \& Mifflin (1985) is

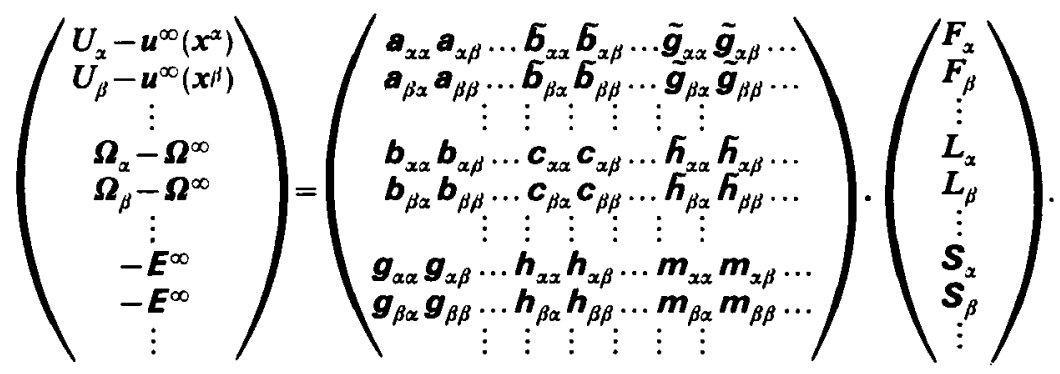

We shall non-dimensionalize all lengths by the sphere radii $a$, and the individual matricies $a, b$, etc. by $6 \pi \mu a^{n}$, where $n=1$ for $\boldsymbol{a}, 2$ for $\boldsymbol{b}$ and 3 for the remaining. The previous authors used the more natural nondimensionalizations given by the Faxén formulae (2.15), but we have used the same $6 \pi \mu$ factor for convenience. With the centre-centre separation of spheres $\alpha$ and $\beta$ denoted by $r$ and the unit vector joining $\alpha$ to $\beta, e_{i}=r_{i} / r$, the symmetry of the two sphere geometry enables us to write

$$
\begin{aligned}
a_{i j}^{\alpha \beta}= & x_{\alpha \beta}^{a} e_{i} e_{j}+y_{\alpha \beta}^{a}\left(\delta_{i j}-e_{i} e_{j}\right) \\
b_{i j}^{\alpha \beta}= & y_{\alpha \beta}^{b} \epsilon_{i j k} e_{k}, \\
c_{i j}^{\alpha \beta}= & x_{\alpha \beta}^{c} e_{i} e_{j}+y_{\alpha \beta}^{c}\left(\delta_{i j}-e_{i} e_{j}\right) \\
g_{i j k}^{\alpha \beta}= & x_{\alpha \beta}^{g}\left(e_{i} e_{j}-\frac{1}{3} \delta_{i j}\right) e_{k}+y_{\alpha \beta}^{g}\left(e_{i} \delta_{j k}+e_{j} \delta_{i k}-2 e_{i} e_{j} e_{k}\right) \\
h_{i j}^{\alpha \beta}= & y_{\alpha \beta}^{h}\left(e_{i} \epsilon_{j k l} e_{l}+e_{j} \epsilon_{i k l} e_{l}\right) \\
m_{i j k l}^{\alpha \beta}= & \frac{3}{2} x_{\alpha \beta}^{m}\left(e_{i} e_{j}-\frac{1}{3} \delta_{i j}\right)\left(e_{k} e_{l}-\frac{1}{3} \delta_{k l}\right) \\
& \quad+\frac{1}{2} y_{\alpha \beta}^{m}\left(e_{i} \delta_{j l} e_{k}+e_{j} \delta_{i l} e_{k}+e_{i} \delta_{j k} e_{l}+e_{j} \delta_{i k} e_{l}-4 e_{i} e_{j} e_{k} e_{l}\right) \\
& +\frac{1}{2} z_{\alpha \beta}^{m}\left(\delta_{i k} \delta_{j l}+\delta_{j k} \delta_{i l}-\delta_{i j} \delta_{k l}+e_{i} e_{j} \delta_{k l}+\delta_{i j} e_{k} e_{l}\right. \\
& \left.\quad+e_{i} e_{j} e_{k} e_{l}-e_{i} \delta_{j l} e_{k}-e_{j} \delta_{i l} e_{k}-e_{i} \delta_{j k} e_{l}-e_{j} \delta_{i k} e_{l}\right)
\end{aligned}
$$

At the level of the approximation in (2.13) and (2.15), the scalar mobility functions are:

$$
\begin{aligned}
& x_{11}^{a}=x_{22}^{a}=1, \quad x_{12}^{a}=x_{21}^{a}=\frac{3}{2} r^{-1}-r^{-3}, \\
& y_{11}^{a}=y_{22}^{a}=1, \quad y_{12}^{a}=y_{21}^{a}=\frac{3}{4} r^{-1}+\frac{1}{2} r^{-3}, \\
& y_{11}^{b}=-y_{22}^{b}=0, \quad y_{12}^{b}=-y_{21}^{b}=-\frac{3}{4} r^{-2}, \\
& x_{11}^{c}=x_{22}^{c}=\frac{3}{4}, \quad x_{12}^{c}=x_{21}^{c}=\frac{3}{4} r^{-3}, \\
& y_{11}^{c}=y_{22}^{c}=\frac{3}{4}, \quad y_{12}^{c}=y_{21}^{c}=-\frac{3}{8} r^{-3}, \\
& x_{11}^{g}=-x_{22}^{g}=0, \quad x_{12}^{g}=-x_{21}^{g}=\frac{9}{4} r^{-2}-\frac{18}{5} r^{-4}, \\
& y_{11}^{g}=-y_{22}^{g}=0, \quad y_{12}^{g}=-y_{21}^{g}=\frac{8}{5} r^{-4}, \\
& y_{11}^{h}=y_{22}^{h}=0, \quad y_{12}^{h}=y_{21}^{h}=-\frac{9}{8} r^{-3}, \\
& x_{11}^{m}=x_{22}^{m}=\frac{9}{10}, \quad x_{12}^{m}=x_{21}^{m}=-\frac{9}{2} r^{-3}+\frac{54}{5} r^{-5}, \\
& y_{11}^{m}=y_{22}^{m}=\frac{9}{10}, \quad y_{12}^{m}=y_{21}^{m}=\frac{9}{9} r^{-3}-\frac{36}{5} r^{-5}, \\
& z_{11}^{m}=z_{22}^{m}=\frac{9}{10}, \quad z_{12}^{m}=z_{21}^{m}=\frac{9}{5} r^{-5} .
\end{aligned}
$$




\section{Appendix B. Equivalence of inverting mobility matrix and summing reflected interactions}

In order to show the equivalence of inverting a mobility matrix and summing reflected interactions, we consider the simple problem of computing the resistance interaction along the line of centres for two spheres. We shall do this at the level of point forces only. We have two spheres, 1 and 2 , and move sphere 2 relative to 1 along the line joining their centres and wish to calculate the total force that must be exerted on particle 1 to keep it fixed. The velocity field created by sphere 2 is simply (all quantities are dimensionless)

$$
u_{i}=\frac{3}{4} J_{i j}\left(x-x^{2}\right) U_{j}^{2} .
$$

Incident on sphere 1 , this velocity field induces a force in sphere 1 , because it is held fixed, given by Faxén's law (2.15a)

$$
F_{i}^{1}=-\frac{3}{4} J_{i j}\left(x^{1}-x^{2}\right) U_{j}^{2}
$$

This induced force, which particle 1 exerts on the fluid, propagates a velocity field

$$
u_{i}=\frac{3}{4} J_{i j}\left(x-x^{2}\right) F_{j}^{1}=-\frac{3}{4} J_{i j}\left(x-x^{2}\right) \frac{3}{4} J_{j k}\left(x^{1}-x^{2}\right) U_{k}^{2},
$$

which in turn induces a force in particle 2 , and so on. In general, the force particle 1 exerts on the fluid owing to the motion of particle 2 is

$$
F_{i}^{1}=-\frac{3}{4} J_{i j}\left(x^{1}-x^{2}\right) U_{j}^{2}-\frac{3}{4} J_{i j}\left(x^{1}-x^{2}\right) \frac{3}{4} J_{j k}\left(x^{2}-x^{1}\right) \frac{3}{4} J_{k l}\left(x^{1}-x^{2}\right) U_{l}^{2}-\ldots
$$

Taking the components along the line of centres, each $J_{i j}$ reduces to $2 / r$, where $r$ is the centre-to-centre spacing.

If we denote the scalar resistance function relating the force on 1 to the velocity of 2 along the line of centres $X_{12}^{A}$, in keeping with the notation of Jeffrey \& Onishi (1984) (note, $x_{12}^{a}$ is the corresponding mobility element).

$$
X_{12}^{A}=-\sum_{n=1}^{\infty}\left(\frac{3}{2 r}\right)^{2 n-1}=-\frac{\frac{3}{2} r^{-1}}{1-\left(\frac{3}{2} r^{-1}\right)^{2}}
$$

As shown by Jeffrey \& Onishi (their equation (1.15)), the scalar resistance functions $X_{12}^{A}, X_{11}^{A}$, etc. can be related to the scalar mobility functions $x_{12}^{a}, x_{11}^{a}$, etc. through inversion of a matrix

$$
\left(\begin{array}{ll}
X_{11}^{A} & X_{12}^{A} \\
X_{12}^{A} & X_{22}^{A}
\end{array}\right)=\left(\begin{array}{ll}
x_{12}^{a} & x_{12}^{a} \\
x_{12}^{a} & x_{22}^{a}
\end{array}\right)^{-1} .
$$

(The full mobility matrix inversion decouples for this component.) Using the point-force approximations from (A 3): $x_{11}^{a}=x_{22}^{a}=1, x_{12}^{a}=x_{21}^{a}=3 / 2 r$, inverting (B 6) gives precisely (B 5) for $X_{12}^{A}$.

This simple example shows the equivalence of inverting the mobility matrix and summing an infinite series of reflections. Whatever elements are used in the mobility matrix - point force, dipole, quadrupole, etc., - all reflected interactions are summed. It is not difficult to carry out a similar sum for the interaction of 3 spheres whose centres lie along the same line. A few terms in the force series equivalent to (B 4) will rapidly show that the inversion of the mobility matrix sums all reflected interactions among all particles. With this simple example one can also start to see 
the nature of screening for fixed particles, because the series equivalent to (B 4) will now have contributions from the third body that are of opposite sign to those for only two particles.

\section{REFERENCES}

ArP, P. A. \& MAson, S. G. 1977 The kinetics of flowing dispersions. VIII. Doublets of rigid spheres (theoretical). J. Colloid Interface Sci. 61, 21-43.

Batchelor, G. K. $1970 a$ The stress system in a suspension of force-free particles. J. Fluid Mech. $41,545-570$.

BATChELOR, G. K. $1970 b$ Slender-body theory for particles of arbitrary cross-section in Stokes flow. J. Fluid Mech. 44, 419-440.

BATCHELOR, G. K. 1976 Brownian diffusion of particles with hydrodynamic interaction. J. Fluid Mech. 74, 1-29.

Batchelor, G. K. \& Green, J. T. 1972 The hydrodynamic interaction of two small freely-moving spheres in a linear flow field. J. Fluid Mech. 56, 375-400.

BEENAKKER, C. W. J. 1984 The effective viscosity of a concentrated suspension of spheres (and its relation to diffusion). Physica 128A, 48-81.

BeEnakker, C. W. J. \& Mazur, P. 1983 Self-diffusion of spheres in a concentrated suspension. Physica 120A, 388-410.

Bossis, G. \& Brady, J. F. 1984 Dynamic simulation of sheared suspensions. I. General Method. J. Chem. Phys. 80, 5141-5154.

Bossis, G. \& BrADY, J. F. 1987 Self diffusion of Brownian particles in concentrated suspensions under shear. J. Chem. Phys. (submitted).

Brady, J. F. \& Bossis, G. 1985 The rheology of concentrated suspensions of spheres in simple shear flow by numerical simulation. J. Fluid Mech. 155, 105-129.

Bretherton, F. P. 1964 Inertial effects on clusters of spheres falling in a viscous fluid. J. Fluid Mech. 20, 401-410.

BRINkman, H. C. 1947 A calculation of the viscous force exerted by a flowing fluid on a dense swarm of particles. Appl. Sci. Res. A 1, 27-34.

Chwang, A. T. \& WU, Y.-T. 1975 Hydromechanics of low-Reynolds-number flow. Part 2. Singularity method for Stokes flows. J. Fluid Mech. 67, 787-815.

Cox, R. G. 1974 The motion of suspended particles almost in contact. Intl J. Multiphase Flow 1 , 343-371.

Durlofsky, L. 1986 Topics in fluid mechanics: I. Flow between finite rotating disks. II. Simulation of hydrodynamically interacting particles in Stokes flow. Ph.D. Thesis, Massachusetts Institute of Technology.

Ganatos, P., Pfeffer, R. \& Weinbaum, S. 1978 A numerical-solution technique for threedimensional Stokes flows, with application to the motion of strongly interacting spheres in a plane. J. Fluid Mech. 84, 79-111.

Happel, J. \& Brenner, H. 1965 Low Reynolds Number Hydrodynamics. Prentice-Hall.

Hocking, L. M. 1964 The behaviour of clusters of spheres falling in a viscous fluid. Part 2. Slow motion theory. J. Fluid Mech. 20, 129-139.

HowelLs, I. D. 1974 Drag due to the motion of a Newtonian fluid through a sparse random array of small fixed rigid objects. J. Fluid Mech. 64, 449-475.

JaYaWEera, K. O. L. F., Mason, B. J. \& Slack, G. W. 1964 The behaviour of clusters of spheres falling in a viscous fluid. Part 1. Experiment. J. Fluid Mech. 20, 121-128.

JEFFREY, D. J. \& ONISHI, Y. 1984 Calculation of the resistance and mobility functions for two unequal rigid spheres in low-Reynolds-number flow. J. Fluid Mech. 139, 261-290.

KIM, S. 1986 Singularity solutions for ellipsoids in low-Reynolds-number flows: with applications to the calculation of hydrodynamic interactions in suspensions of ellipsoids. Intl J. Multiphase Flow 12, 469-491.

Krm, S. \& Mifflin, R. T. 1985 The resistance and mobility functions of two equal spheres in low-Reynolds-number flow. Phys. Fluids 28, 2033-2045. 
Kynch, G. J. 1959 The slow motion of two or more spheres through a viscous fluid. J. Fluid Mech. 5, 193-208.

LadyzhenskayA, O. A. 1963 The Mathematical Theory of Viscous Incompressible Flow. Gordon \& Breach.

Mazur, P. \& SaARloos, W. van 1982 Many-sphere hydrodynamic interactions and mobilities in a suspension. Physica 115A, 21-57.

Youngren, G. K. \& ACRIvos, A. 1975 Stokes flow past a particle of arbitrary shape: a numerical method of solution. J. Fluid Mech. 69, 377-403. 